\title{
Atomic and molecular gas in the merger galaxy NGC 1316 (Fornax A) and its environment
}

\author{
C. Horellou ${ }^{1}$, J. H. Black ${ }^{1}$, J. H. van Gorkom² ${ }^{2}$ F. Combes ${ }^{3}$, J. M. van der Hulst ${ }^{4}$, and V. Charmandaris ${ }^{5}$ \\ 1 Onsala Space Observatory, Centre for Astrophysics \& Space Science, Chalmers University of Technology, \\ 43992 Onsala, Sweden \\ e-mail: jblack@oso.chalmers.se \\ 2 Astronomy Department, Columbia University, 538 W 120 Street, New York, NY 10027, USA \\ e-mail: jvangork@astro.columbia.edu \\ 3 Observatoire de Paris, DEMIRM, 61 avenue de l'Observatoire, 75014 Paris, France \\ e-mail: Francoise.Combes@obspm.fr \\ ${ }^{4}$ Kapteyn Institute, Department of Astronomy, Postbus 800, 9700 AV Groningen, The Netherlands \\ e-mail: j.m.van.der.hulst@astro.rug.nl \\ ${ }^{5}$ Cornell University, Astronomy Department, 106 Space Sciences Building, Ithaca, NY 14853, USA \\ e-mail: vassilis@astro.cornell.edu
}

Received 4 May 2001 / Accepted 12 July 2001

\begin{abstract}
We present and interpret observations of atomic and molecular gas toward the southern elliptical galaxy NGC 1316 (Fornax A), a strong double-lobe radio source with a disturbed optical morphology that includes numerous shells and loops. The ${ }^{12} \mathrm{CO}(1-0),{ }^{12} \mathrm{CO}(2-1)$, and Hi observations were made with SEST and the VLA. $\mathrm{CO}$ emission corresponding to a total molecular hydrogen mass of $\sim 5 \times 10^{8} M_{\odot}$ was detected towards the central position as well as northwest and southeast of the nucleus in the regions of the dust patches. The origin of that gas is likely external and due to accretion of one or several small gas-rich galaxies. Hi was not detected in the central region of NGC 1316, but $\sim 2 \times 10^{7} M_{\odot}$ of atomic gas was found towards the giant His region discovered by Schweizer (1980) located 6.7 (or $36.2 \mathrm{kpc}$ ) from the nucleus. Hi was also found at three other locations in the outer part of NGC 1316. The Hi distributions and kinematics of the two nearby spiral companions of NGC 1316, NGC 1317 (a barred galaxy to the north) and NGC 1310 (to the west) could be studied. Both galaxies have unusually small $\mathrm{HI}$ disks that may have been affected by ram-pressure stripping.
\end{abstract}

Key words. galaxies: ellipticals - individual (NGC 1316, NGC 1317, NGC 1310) - galaxies: clusters: Fornax cluster - radio lines: galaxies

\section{Introduction}

Over the past fifteen years, evidence for the existence of a complex, multi-phase interstellar medium in elliptical galaxies has accumulated. Most of the gas in ellipticals is hot $\left(10^{7} \mathrm{~K}\right)$ and radiates at X-ray wavelengths, but there is also a cooler component of $\mathrm{HI}, \mathrm{CO}$, ionized gas and dust (e.g. Bregman et al. 1992). The origin and fate of the gas are still debated, but it seems that the hot gas is associated with the spheroid, whereas the atomic and molecular gas as traced by the $\mathrm{CO}$ characterize a prominent disk (e.g. van Gorkom \& Schiminovich 1997). The detection rates of $\mathrm{HI}$ or $\mathrm{CO}$ are significantly higher among ellipticals with peculiar features (such as tidal tails, loops, shells, dust lanes) than in the whole class of ellipticals, which suggests

Send offprint requests to: C. Horellou, e-mail: horellou@oso.chalmers.se that the gas is of external origin and due to accretion of smaller companions and/or to major mergers.

NGC 1316 (Fornax A, Arp 154, PKS 0320-37) on the outskirts of the Fornax cluster presents several characteristics of a merger remnant. The disturbed outer morphology with numerous shells and loops was noted by Matthews et al. (1964) and Arp (1964). The galaxy was extensively studied in the optical by Schweizer (1980, hereafter S80) and Schweizer (1981) who classified it as a D-type galaxy with an elliptical-like spheroid surrounded by a large envelope. The brightness profile approximately obeys the $r^{1 / 4}$ law. The galaxy has an inner dust lane of length $\sim 2^{\prime}$ oriented close to the minor axis. S80 also reported the discovery of a giant HiI region (hereafter denoted SH2) 6!7 from the nucleus, and the existence of an inner disk of ionized gas probably associated with the dust lane and extending out to $54^{\prime \prime}$. The disk is in rapid rotation 


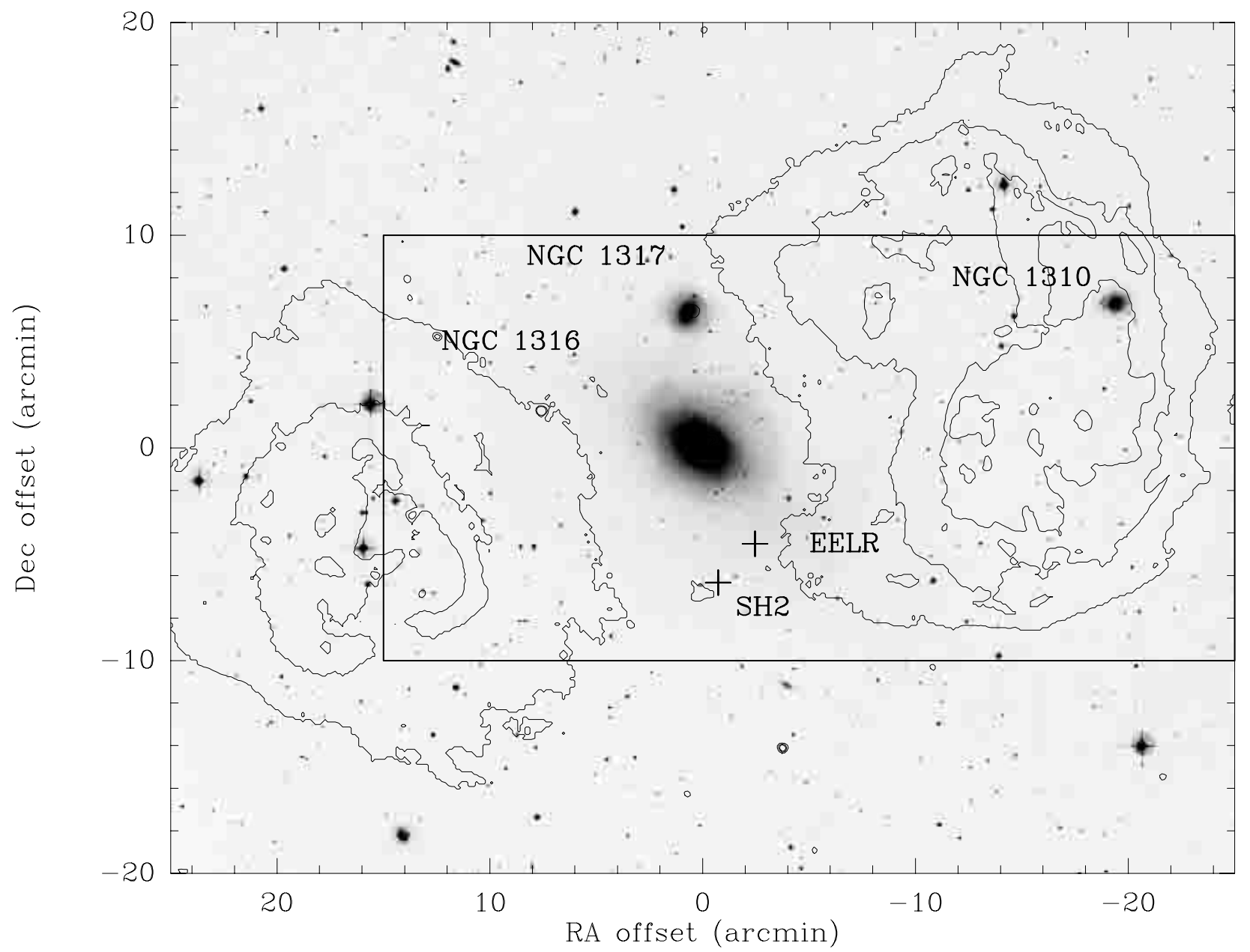

Fig. 1. Radio continuum contour map at $\lambda 20 \mathrm{~cm}$ of Fomalont et al. (1989) superimposed on an optical image taken from the Digitized Sky Survey. The resolution of the radio continuum map is $14^{\prime \prime}$. The two neighbor galaxies, NGC 1317 and NGC 1310 , and the location of the extended emission line region EELR and the giant Hir region SH2 in the southern tidal loop called $L_{1}$ by Schweizer (1980) are indicated. NGC 1310 is located in the foreground of the western radio lobe. The black rectangle shows the size of the image displayed in Fig. 2.

$\left(v \sin i \sim 350 \mathrm{kms}^{-1}\right.$ ). To explain those features and the lack of a nearby perturber, S80 suggested that NGC 1316 accreted one or several gas-rich companion galaxies about $4 \times 10^{8}-2 \times 10^{9}$ years ago. Mackie \& Fabbiano (1998) reported the discovery of an extended emission-line region (hereafter denoted EELR) located in a tidal tail 5:3 from the nucleus and suggested on the basis of its position and extreme size that it is related to the merger process.

NGC 1316 is the third nearest strong radio galaxy in the sky after NGC 5128 (Centaurus A) and M 87 (Virgo A) (see Fig. 1). The radio emission arises in two giant lobes separated by $33^{\prime}$ and located mainly outside the optical galaxy, at a position angle of $\sim 110^{\circ}$ (Wade 1961; Ekers et al. 1983; Fomalont et al. 1989). In the central arcmin a bent radio jet abruptly terminates at the location of a dust lane, which may have deflected it (Geldzahler $\&$ Fomalont 1984). The nucleus is a bright UV source, unresolved by HST (Fabbiano et al. 1994).

A study of the stellar kinematics of NGC 1316 showed that the galaxy is rotating rapidly around the minor axis at a position angle of $\sim 140^{\circ}$ (Bosma et al. 1985). More recently Arnaboldi et al. (1998) measured radial velocities of 43 planetary nebulae in the outer parts of NGC 1316 to determine the distribution of angular momentum. They derived a constant velocity $v=110 \mathrm{kms}^{-1}$ along the major axis of the galaxy in the radial range $R=50$ $200^{\prime \prime}$. They also probed the kinematics of the inner region $\left(R<80^{\prime \prime}\right)$ using absorption line spectra. They confirmed the alignment between the photometric and the kinematic minor axes but also found evidence for a disturbed kinematical pattern beyond $R \sim 30^{\prime \prime}$.

X-ray emission from NGC 1316 has been observed with several instruments. The $L_{\mathrm{X}} / L_{B}$ ratio of NGC 1316 is low compared to that of E and S0 galaxies (Einstein observations by Fabbiano et al. 1992; Kim et al. 1992), but in the same range as that of dynamically young ellipticals which may have experienced mergers (Fabbiano \& Schweizer 1995; see also Sansom et al. 2000).

In order to place NGC 1316 into the merger sequence as discussed by Hibbard \& van Gorkom (1996) it is necessary to know the distribution of the cool gas, both the neutral atomic hydrogen and the molecular 
hydrogen as traced by the CO. Sage \& Galleta (1993) detected ${ }^{12} \mathrm{CO}(1-0)$ line emission at two positions along the inner dust lane and with kinematics similar to that of the ionized gas. In this paper, we present more extensive and more sensitive maps of the ${ }^{12} \mathrm{CO}(1-0)$ and the first ${ }^{12} \mathrm{CO}(2-1)$ observations in the central part of NGC 1316 as well as a search for $\mathrm{CO}$ emission toward the outer HII complexes. We also present results from a VLA search for Hi gas in NGC 1316 and its surroundings.

The paper is organized as follows: we first describe the $\mathrm{HI}$ and $\mathrm{CO}$ observations, then discuss the Hi results for the companion galaxies NGC 1317 and NGC 1310. We then focus on the HI and $\mathrm{CO}$ results for NGC 1316 and the implications for the merger picture.

\section{Observations and data reduction}

\subsection{HI observations}

The Hi observations consist of data collected with the Very Large Array (VLA) ${ }^{1}$ in its spectral-line mode. The galaxy was observed in the hybrid DnC configuration: the $1.3 \mathrm{~km}$ D-array configuration with the antennas of the north arm in the $3 \mathrm{~km}$ C-array configuration to improve the NorthSouth angular resolution, thus compensating for the low declination of NGC 1316. The observations consisted of two runs of 5 hours each.

A $6.25 \mathrm{MHz}$ bandwidth was used, corresponding to a total velocity range of $1300 \mathrm{~km} \mathrm{~s}^{-1}$. On-line Hanning smoothing has been applied to the data after which every other channel was discarded, leaving a set of 31 independent channels centered at $1774 \mathrm{~km} \mathrm{~s}^{-1}$ and spaced by $41.7 \mathrm{~km} \mathrm{~s}^{-1}$. The observing parameters are summarized in Table 1.

The data were inspected, calibrated, and maps were produced using NRAO's Astronomical Image Processing System (AIPS). The continuum was estimated from channels free of line emission and was subtracted in the UV plane. Line channel maps were made using uniform weighting with a ROBUST factor of 1 which, after some experimentation, was found to give the best combination of sensitivity and spatial resolution. The channel maps were CLEANed and a correction for primary beam attenuation was applied to the entire data cube. Maps of the atomic hydrogen column density and velocity field were produced. The maps were not smoothed. Only pixels above a flux cut-off of $1.5 \mathrm{mJy} /$ beam $(2.5 \sigma)$ were included in the summation of the channel maps.

The data were taken in 1985 by van Gorkom, Ekers, Schweizer and Wrobel. Due to limitations in on-line and off-line computing power only 25 antennas and one polarization (RR) could be used. The data were fully reduced by van Gorkom, but never published. Although NGC 1310, NGC 1317 and the HiI region were unambiguously detected, the quality of the data reduction was

1 The VLA is a facility of the U.S. National Radio Astronomy Observatory, which is operated by Associated Universities Inc. under contract with the U.S. National Science Foundation.
Table 1. VLA observing parameters.

\begin{tabular}{ll}
\hline Phase center $(\mathrm{B} 1950)$ & $03^{\mathrm{h}} 20^{\mathrm{m}} 47^{\mathrm{s}} .2 ;-37^{\circ} 23^{\prime} 08^{\prime \prime} .2$ \\
Velocity center & $1774 \mathrm{~km} \mathrm{~s}^{-1}$ (heliocentric) \\
Primary beam $(F W H M)$ & $30^{\prime}$ \\
Flux calibrator & $3 \mathrm{C} 48$ \\
Phase calibrator & $0332-403$ \\
Bandpass calibrator & $3 \mathrm{C} 48$ \\
Array & DnC \\
Date & 19,21 Oct. 1985 \\
Synthesized beam & \\
- FW $M$ : major $\times$ minor axis & $52^{\prime \prime} \times 41^{\prime \prime}$ \\
- Position angle & $+2^{\circ}$ \\
Bandwidth & $6.25 \mathrm{MHz}$ \\
Number of channels & 31 \\
Channel separation & $41.7 \mathrm{~km} \mathrm{~s}^{-1}$ \\
Time on source & $6 \mathrm{~h} 51 \mathrm{~min}^{*}$ \\
Noise level $(1 \sigma)^{*}$ & \\
- Flux density & $0.6 \mathrm{mJy} \mathrm{beam} \mathrm{ch}^{-1}$ \\
- Column density & $1.3 \times 10^{19} \mathrm{~cm}^{-2} \mathrm{beam}^{-1} \mathrm{ch}^{-1}$ \\
- Hi mass & $2 \times 10^{6} M_{\odot} \mathrm{beam}^{-1} \mathrm{ch}^{-1}$ \\
\hline
\end{tabular}

* Noise level in the continuum-subtracted maps before primary beam correction.

poor. This paper shows that reprocessing of ancient (but venerable) data is very worthwhile, especially when a large spectral dynamic range is needed, because there have been major improvements in software such as the UVLIN program within AIPS and far better weighting schemes in the imaging tasks.

\subsection{CO observations}

The observations were done with the $15 \mathrm{~m}$ Swedish-ESO Submillimeter Telescope (SEST) ${ }^{2}$ on La Silla, Chile, in two sessions on 1999 December 7-13 and in 2001 January. We used the IRAM dual receiver to observe simultaneously at the frequencies of the ${ }^{12} \mathrm{CO}(1-0)$ and ${ }^{12} \mathrm{CO}(2-1)$ lines. At $115 \mathrm{GHz}$ the half-power beamwidth of the telescope is $43^{\prime \prime}$, which corresponds to a linear scale of about $3.9 \mathrm{kpc}$ at the adopted distance of $18.6 \mathrm{Mpc}$. The $\mathrm{CO}(2-1)$ map has a resolution of $22^{\prime \prime}$ (or $\sim 2 \mathrm{kpc}$ ). This is the highest resolution map of molecular gas in NGC 1316 available to date. A balanced on-off beam-switching mode was used. The pointing was regularly checked on nearby $\mathrm{SiO}$ masers. The temperature scale used throughout this article is the main-beam temperature $T_{\mathrm{mb}}=T_{\mathrm{A}}^{*} / \eta_{\mathrm{mb}}$. The main-beam efficiency of SEST is $\eta_{\mathrm{mb}}=0.7$ at $115 \mathrm{GHz}$ and 0.5 at $230 \mathrm{GHz}$. The data were analyzed using the software CLASS. The spectra were smoothed to a final velocity resolution of $\sim 14.5 \mathrm{~km} \mathrm{~s}^{-1}$. Only first-order baselines were subtracted.

${ }^{2}$ The SEST is operated jointly by the European Southern Observatory (ESO) and the Swedish National Facility for Radio Astronomy, Chalmers Centre for Astrophysics and Space Science. 
Table 2. Basic parameters of the galaxies and outer Hiı regions.

\begin{tabular}{|c|c|c|c|c|c|c|}
\hline & Note & NGC 1316 & NGC 1317 & NGC 1310 & SH2 & EELR \\
\hline RA(1950) & (1) & $03^{\mathrm{h}} 20^{\mathrm{m}} 47.2^{\mathrm{s}}$ & $03^{\mathrm{h}} 20^{\mathrm{m}} 49.8^{\mathrm{s}}$ & $03^{\mathrm{h}} 19^{\mathrm{m}} 08.8^{\mathrm{s}}$ & $03^{\mathrm{h}} 20^{\mathrm{m}} 43.5^{\mathrm{s}}$ & $03^{\mathrm{h}} 20^{\mathrm{m}} 34.8^{\mathrm{s}}$ \\
\hline $\operatorname{DEC}(1950)$ & (1) & $-37^{\circ} 23^{\prime} 08^{\prime \prime}$ & $-37^{\circ} 16^{\prime} 50.9^{\prime \prime}$ & $-37^{\circ} 16^{\prime} 42.3^{\prime \prime}$ & $-37^{\circ} 29^{\prime} 28^{\prime \prime}$ & $-37^{\circ} 27^{\prime} 39^{\prime \prime}$ \\
\hline$v_{\text {helio }}\left[\mathrm{km} \mathrm{s}^{-1}\right]$ & (2) & 1783 & 1948 & 1739 & 1690 & \\
\hline$D[\mathrm{Mpc}]$ & (3) & 18.6 & 18.6 & 18.6 & 18.6 & 18.6 \\
\hline Type & (4) & .LXSOP. & .SXR1.. & .SAS5*. & & \\
\hline Size & (5) & $12^{\prime} \times 8^{\prime} .5$ & $2^{\prime} .75 \times 2.4$ & $2^{\prime} .0 \times 1^{\prime} .55$ & $1^{\prime \prime} 5 \times 3^{\prime \prime} .5$ & $81^{\prime \prime} \times 27^{\prime \prime}$ \\
\hline $\mathrm{PA}\left[{ }^{\circ}\right]$ & (5) & 50 & 78 & 95 & & $\sim 45$ \\
\hline$B_{\mathrm{T}}^{0}[\mathrm{mag}]$ & (6) & 9.4 & 11.81 & 12.56 & & \\
\hline$L_{B}\left[L_{\odot}\right]$ & (6) & $9.35 \times 10^{10}$ & $1.02 \times 10^{10}$ & $5.1 \times 10^{9}$ & & \\
\hline$L_{\mathrm{FIR}}\left[L_{\odot}\right]$ & (7) & $2.05 \times 10^{9}$ & $2.45 \times 10^{9}$ & $7.7 \times 10^{8}$ & & \\
\hline$M(\mathrm{HI})\left[M_{\odot}\right]$ & (8) & $<10^{8}$ & $2.7 \times 10^{8}$ & $4.8 \times 10^{8}$ & $1.9 \times 10^{7}$ & $<3 \times 10^{6}$ \\
\hline$M\left(\mathrm{H}_{2}\right)\left[M_{\odot}\right]$ & (9) & $\sim 5 \times 10^{8}$ & $3.1 \times 10^{8}$ & $<0.2 \times 10^{8}$ & $<7 \times 10^{6}$ & $<7 \times 10^{6}$ \\
\hline$v_{\mathrm{H} \mathrm{I}}, \Delta v_{\mathrm{H} \text { I }}\left[\mathrm{km} \mathrm{s}^{-1}\right]$ & (8) & & 1960,132 & 1827,144 & $1690, \leq 42$ & $1750, \leq 42$ \\
\hline$F_{\mathrm{H} \text { I }}\left[\mathrm{Jy} \mathrm{km} \mathrm{s}^{-1}\right]$ & (8) & $<0.1$ & $3.3 \pm 0.5$ & $5.9 \pm 1.3$ & & \\
\hline$v_{\mathrm{CO}}, \Delta v_{\mathrm{CO}}\left[\mathrm{km} \mathrm{s}^{-1}\right]$ & (9) & & 1948,84 & & & \\
\hline
\end{tabular}

Notes: (1) NGC 1316: Schweizer (1981); NGC 1317: da Costa et al. (1998); NGC 1310: Loveday (1996); SH2 and EELR: calculated from the offsets relative to the central position given by Mackie \& Fabbiano (1998).

(2) NGC 1316: Arnaboldi et al. (1998); NGC 1317: Horellou et al. (1995); NGC 1310: RC3; SH2: this work.

(3) Distance estimate for NGC 1365 based on HST measurements of cepheid variable stars (Madore et al. 1999). At that distance, $1^{\prime}$ corresponds to $5.4 \mathrm{kpc}$. That distance was adopted for all the systems discussed here.

(4) RC3.

(5) For the three galaxies: optical diameters at the 25 mag $\operatorname{arcsec}^{-2}$ isophote taken from RC3; SH2: Schweizer (1980); EELR: Mackie \& Fabbiano (1998).

(6) RC3.

(7) Derived from the fluxes at 60 and $100 \mu \mathrm{m}$ listed in the IRAS catalogue using the relation: $L_{\mathrm{FIR}}=3.94 \times 10^{5} D^{2}\left[2.58 f_{60}+f_{100}\right]$ where $D$ is the distance in Mpc, $f_{60}$ and $f_{100}$ are the fluxes in Jy, and the far-infrared luminosity $L_{\mathrm{FIR}}$ is in solar luminosities. (8) This work.

(9) NGC 1316, SH2 and EELR: this work. H2 mass of NGC 1317 and NGC 1310: Horellou et al. (1995) after correction for the distance adopted here.

\subsection{Determination of gas masses}

The integrated Hi masses are calculated using:

$M_{\mathrm{HI}}\left[M_{\odot}\right]=2.356 \times 10^{5} D_{\mathrm{Mpc}}^{2} \int S_{\mathrm{HI}} \mathrm{d} v$

where $D_{\mathrm{Mpc}}$ is the distance in Mpc and $\int S_{\mathrm{HI}} \mathrm{d} v$ is the integrated Hi flux in $\mathrm{Jy} \mathrm{km} \mathrm{s}^{-1}$.

Hi column densities can be derived from the observed integrated intensities by using the relation:

$N_{\mathrm{HI}}=\frac{110.4 \times 10^{3}}{a b} \int S_{\mathrm{HI}} \mathrm{d} v$

where $N_{\mathrm{HI}}$ is the column density in $10^{19} \mathrm{~cm}^{-2}$ per beam per channel, and $a$ and $b$ are the diameters of the synthesized beam at full width half maximum in arcsec.

From the ${ }^{12} \mathrm{CO}(1-0)$ line intensities we can estimate the mass of molecular gas $\left(\mathrm{H}_{2}\right)$ within the $43^{\prime \prime}$ SEST beam:

$M\left(\mathrm{H}_{2}\right)\left[M_{\odot}\right]=1.25 \times 10^{5}\left(\theta / 43^{\prime \prime}\right)^{2} I_{\mathrm{mb}}(\mathrm{CO}) D_{\mathrm{Mpc}}^{2}$

where $\theta$ is the half-power beam width of the telescope and $I_{\mathrm{mb}}=\int T_{\mathrm{mb}} \mathrm{d} v$ in $\mathrm{K} \mathrm{km} \mathrm{s}^{-1}$. To be consistent with previous papers, we have used a conversion factor $X=N\left(\mathrm{H}_{2}\right) / I(\mathrm{CO})$ of $2.3 \times 10^{20} \mathrm{~mol} \mathrm{~cm}^{-2}\left(\mathrm{~K} \mathrm{~km} \mathrm{~s}^{-1}\right)^{-1}$
(Strong et al. 1988). However, use of the Galactic CO$\mathrm{H}_{2}$ conversion factor may lead to an underestimate of the molecular gas mass for elliptical galaxies with a significant amount of cold dust (Wiklind et al. 1995).

\section{Atomic hydrogen}

Figure 2 shows the Hi field of interest around NGC 1316 superimposed on an optical image from the Digitized Sky Survey. Hi emission was found in the two companion galaxies NGC 1317 (to the north) and NGC 1310 (to the west). No HI was detected in the main body of NGC 1316 to a limiting mass of $\sim 10^{8} M_{\odot}$ but some was found at four locations in the outer part of the galaxy. One of these is associated with the outer HII region SH2. The other is coincident with the extended emission line region EELR. The two other Hi concentrations to the west and northwest of NGC 1316 are located at the edge of an optical plateau between the area of X-ray emission as observed by ROSAT (Mackie \& Fabbiano 1998) and the extended western radio lobe (see Fig. 1). The mass of each of these Hi concentrations is $\sim 2 \times 10^{7} M_{\odot}$. We will defer discussion of these Hi features and the lack of Hi emission in the main spheroid of NGC 1316 until later and first discuss the Hi properties of NGC 1317 and NGC 1310. 


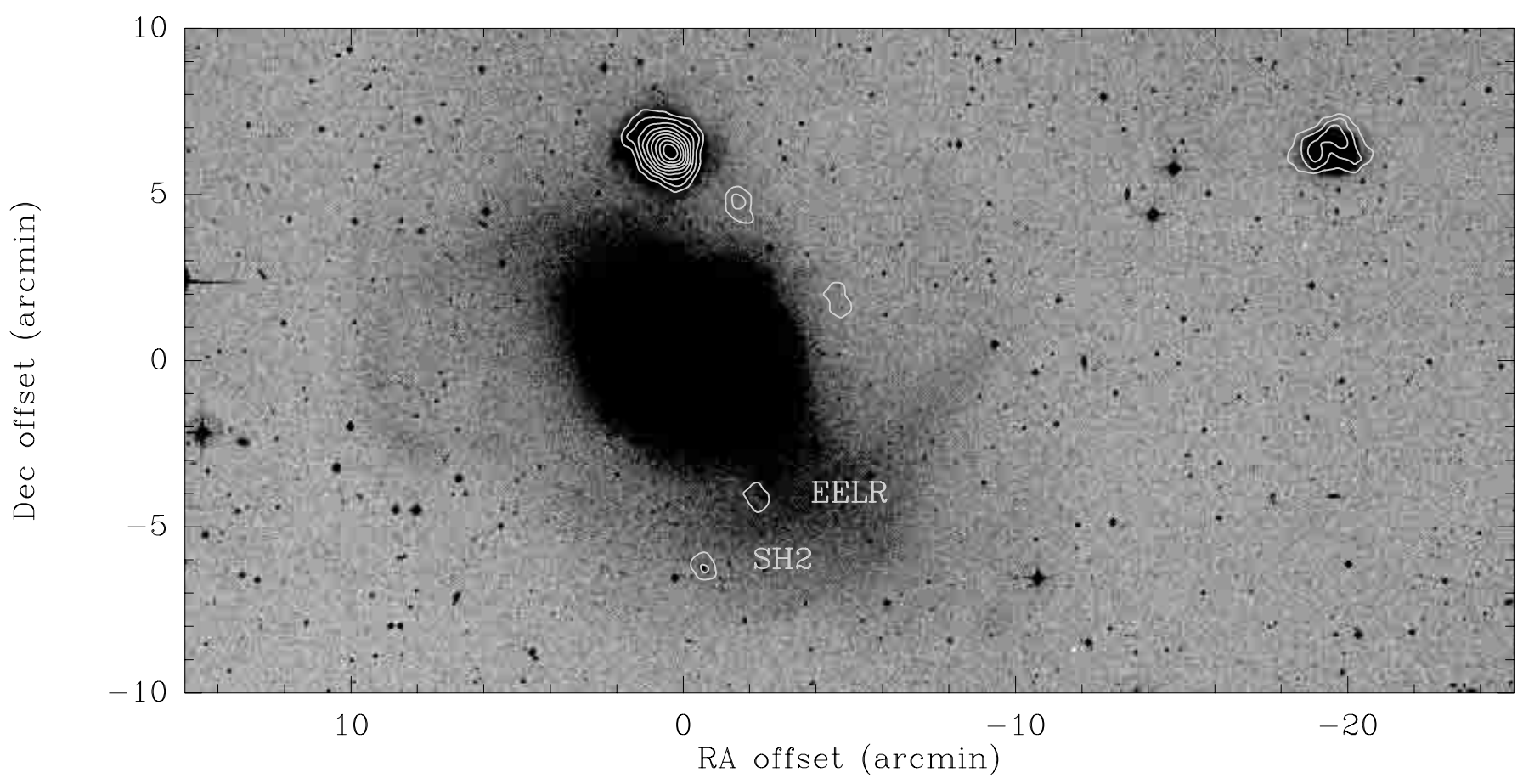

Fig. 2. Integrated Hi map overlaid on an optical image from the Digitized Sky Survey. The contour levels are $n \times 5.2 \times 10^{19} \mathrm{~cm}^{-2}$. The first contour is at the $4 \sigma$ level. The two giant Hir regions to the south, SH2 and EELR, are detected. No primary beam correction has been applied to this map.
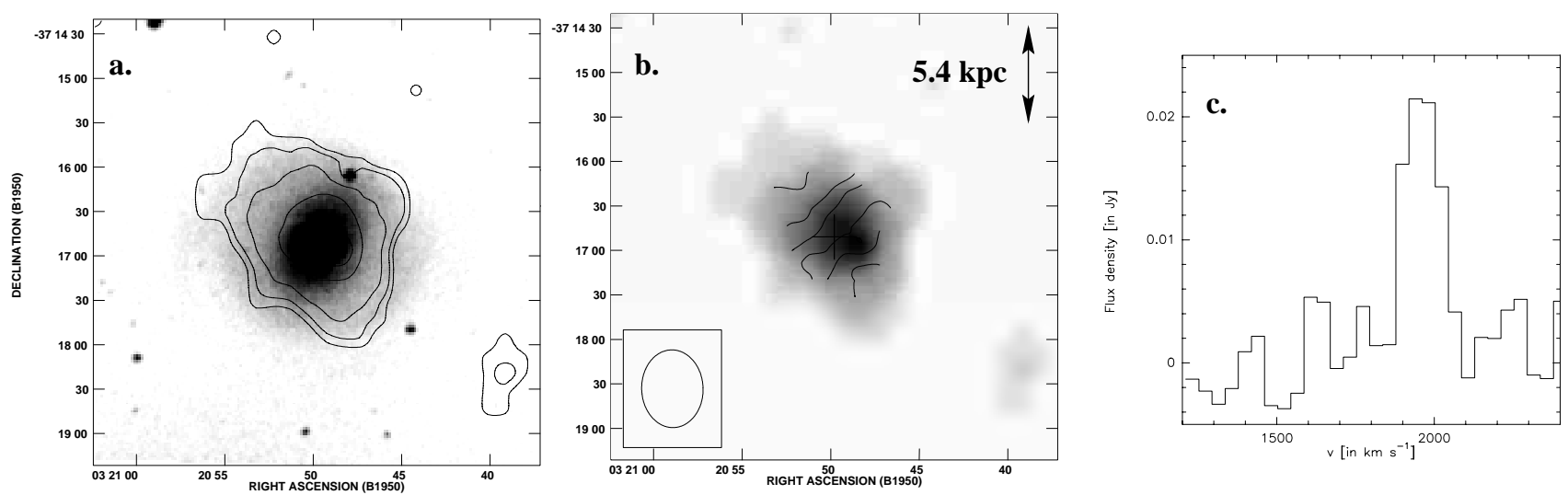

Fig. 3. a) Hi integrated intensity map of NGC 1317 overlaid on a grey scale optical image from the Digitized Sky Survey. A correction for the primary beam has been applied. The levels are $(4,8,16,31,47) \times 10^{19} \mathrm{~cm}^{-2}$. The first contour corresponds to the $2 \sigma$ level. b) Hi velocity field overlaid on a grey scale image of the Hi integrated intensity. Contours of constant heliocentric radial velocity begin at $v=1910 \mathrm{~km} \mathrm{~s}^{-1}$ in the northeast and increase in steps of $20 \mathrm{~km} \mathrm{~s}^{-1}$ toward the southwest. The cross marks the position of the optical center of NGC 1317. c) Integrated Hi line profile derived from the observations.

\subsection{The neighbor galaxies NGC 1317 and NGC 1310}

NGC 1317 is a small barred galaxy north of NGC 1316 . The two galaxies have a projected separation on the sky of $6.3(34 \mathrm{kpc})$ and a radial velocity difference of $+165 \mathrm{~km} \mathrm{~s}^{-1}$. An inner bar perpendicular to the primary bar is visible in the optical images of Schweizer (1980) and Wozniak et al. (1995). A ring of star formation is seen in the optical images and in the ultraviolet image obtained with the Ultraviolet Imaging Telescope. The primary bar is "fat" $\left(\sim 60^{\prime \prime} \times 80^{\prime \prime}\right)$. The secondary bar has a position angle of $\sim 60^{\circ}$ and a total length of $\sim 14^{\prime \prime}$. S 80 assigns
NGC 1317 a late $\mathrm{SB}(\mathrm{rs})$ a or early $\mathrm{SB}(\mathrm{rs}) \mathrm{b}$ type in the Hubble classification.

Figure 3 presents in form of isocontours the atomic hydrogen distribution and the velocity field in NGC 1317 derived from the observations. Also shown is the global Hi spectrum. The channel maps are shown in Fig. 4. The Hi distribution is more extended than the beam size and is elongated in the direction of the minor axis of the galaxy. The gas is clearly rotating, with the south-west side receding. The velocity and the linewidth of the global Hi spectrum agree with those of the ${ }^{12} \mathrm{CO}(1-0)$ line detected by Horellou et al. (1995) (see Table 2). NGC 1317 


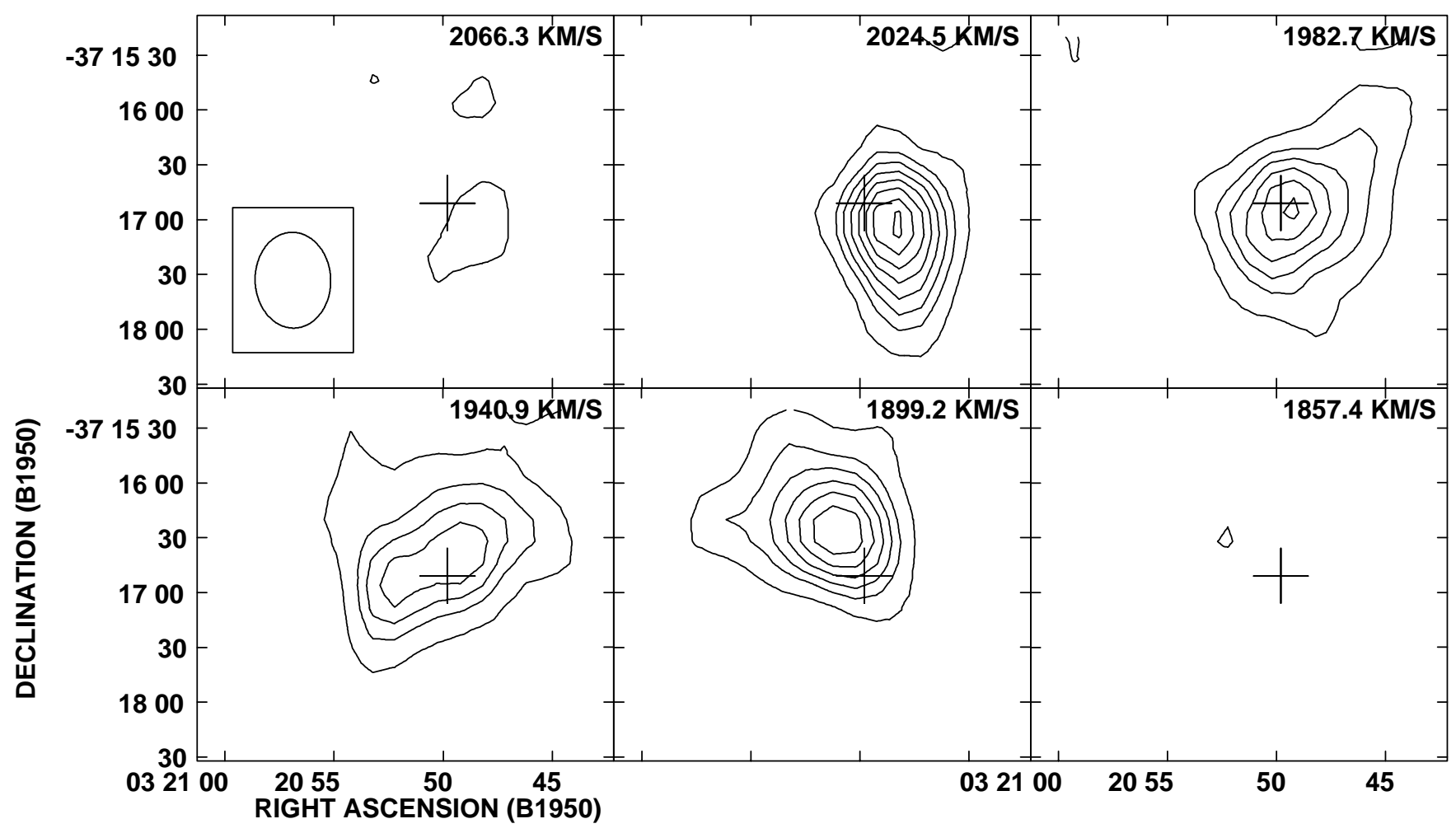

Fig. 4. The Hi line emission of NGC 1317 displayed as channel maps running from $2066 \mathrm{~km} \mathrm{~s}^{-1}$ (top left panel) to $1857 \mathrm{~km} \mathrm{~s}{ }^{-1}$ (bottom right panel). North is up and east is left. The levels are 1.5, 3, 4, 5, 6, 7, 8, $9 \mathrm{mJy} / \mathrm{beam}$. The first contour corresponds to the $2 \sigma$ level. The cross marks the position of the optical center of NGC 1317.
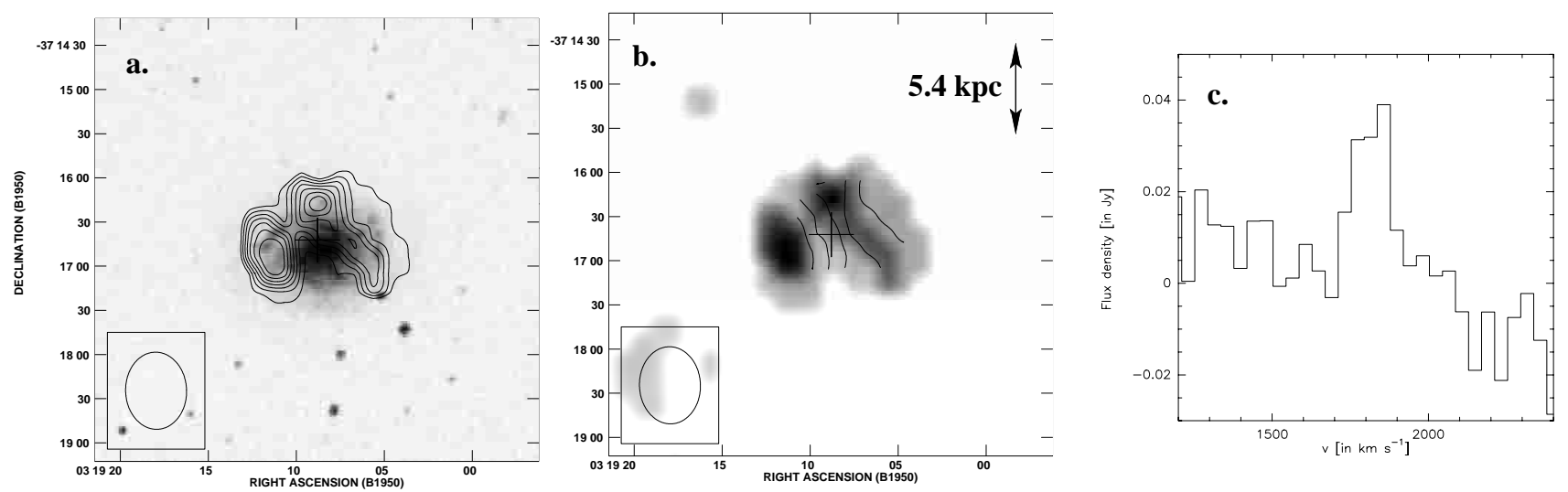

Fig. 5. a) Hi integrated intensity map of NGC 1310 overlaid on a grey scale optical image from the Digitized Sky Survey. A correction for the primary beam has been applied. The levels range from 21 to $52 \times 10^{19} \mathrm{~cm}^{-2}$ by step of $5.2 \times 10^{19} \mathrm{~cm}^{-2}$. The first contour corresponds to the $4 \sigma$ level. b) Hi velocity field overlaid on a grey scale image of the Hi integrated intensity. Contours of constant heliocentric radial velocity begin at $v=1770 \mathrm{~km} \mathrm{~s}^{-1}$ in the east and increase in steps of $20 \mathrm{kms} \mathrm{s}^{-1}$ toward the west. The cross marks the position of the optical center of NGC 1310. c) Integrated Hi line profile derived from the observations.

has comparable amounts of molecular and atomic gas $\left(\sim 3 \times 10^{8} M_{\odot}\right)$.

The HI disk of NGC 1317 is small compared to the optical extent. The $\mathrm{HI}$ isophote corresponding to a column density of $10^{20} \mathrm{~cm}^{-2}$ crosses the major axis of the galaxy at a radius of about $55^{\prime \prime}$ whereas the optical isophote at $25 \mathrm{mag} \operatorname{arcsec}^{-2}$ is found at $82 . .5$. The ratio of the extent of the Hi disk to that of the optical image is therefore 0.7 , which places NGC 1317, if classified as an Sab galaxy, in the group of galaxies with an HI distribution strongly affected by the environment (group III of Cayatte et al. 1994 for Virgo galaxies). Cayatte et al. (1994) showed that those Virgo galaxies with a truncated Hi disk have a normal central Hi surface density average as compared to field galaxies and concluded that the galaxies (all located close to the cluster core) have been affected by ram-pressure sweeping. NGC 1317 has a central Hi surface density averaged over half the optical radius of $\sim 3 \times 10^{20} \mathrm{~cm}^{-2}$, which is lower 


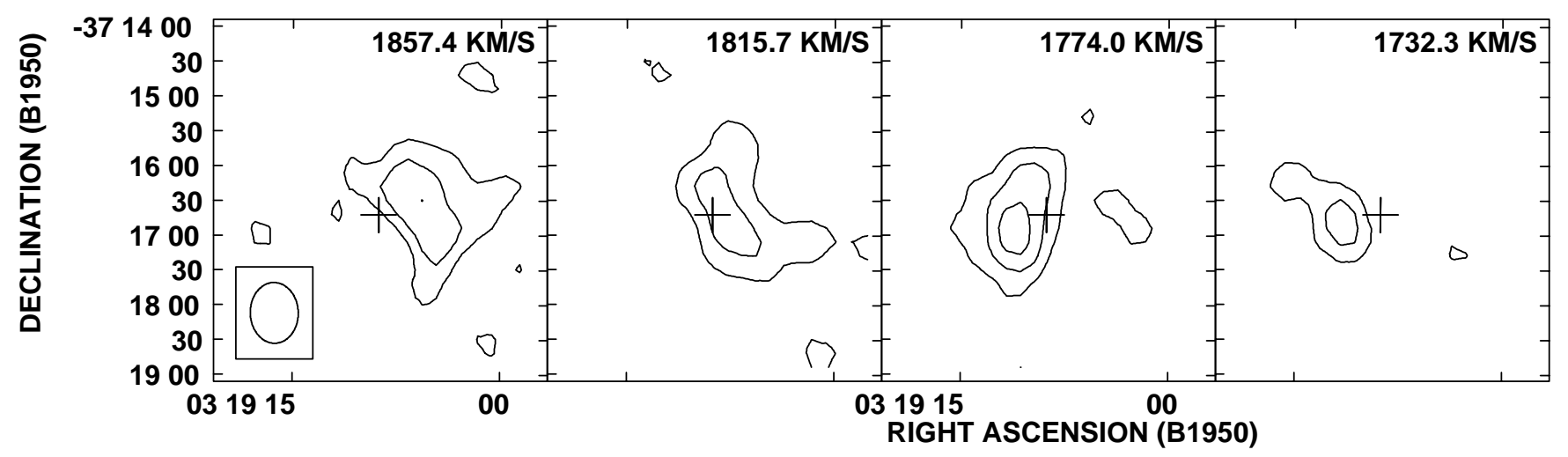

Fig. 6. The Hi line emission of NGC 1310 displayed as channel maps running from $1857 \mathrm{~km} \mathrm{~s}^{-1}$ (left panel) to $1732 \mathrm{~km} \mathrm{~s}^{-1}$ (right panel). North is up and east is left. The levels are 4,8 and $12 \mathrm{mJy} / \mathrm{beam}$. The first contour corresponds to the $2 \sigma$ level. The cross marks the position of the optical center of NGC 1310.

than that of Sab galaxies in the comparison sample of Cayatte et al. $\left(5.0 \pm 1.2 \times 10^{20} \mathrm{~cm}^{-2}\right)$, but in the range of values found for galaxies of earlier types ( $\mathrm{S} 0, \mathrm{~S} 0 / \mathrm{a}$, Sa have $3.4 \pm 0.9 \times 10^{20} \mathrm{~cm}^{-2}$ ). Given the uncertainty in the morphological type and the fact that we are discussing an individual galaxy, it is difficult to draw any firm conclusion about the central Hi density of NGC 1317. Nevertheless, it is clear that the HI disk is small and that NGC 1317 has a low Hi mass for its optical size and morphological type. We calculate an Hi deficiency of .64 in the log, or a factor of 4.4 using the average value for isolated Sab galaxies observed by Haynes \& Giovanelli (1984) and the definition: HI def $=\log \left(M(\mathrm{HI}) / D^{2}\right)_{\mathrm{NGC} 1317}-\log \left(M(\mathrm{HI}) / D^{2}\right) c s$ where $c s$ stands for control sample. Although NGC 1316 is not located in the center of the Fornax cluster, it does dominate its corner of the cluster and is a source of X-ray emission. It is possible that NGC 1317 has passed through the X-ray halo of NGC 1316 and been stripped of some of its atomic gas.

NGC 1310 is an almost face-on spiral fortuitously positioned between us and the western radio lobe of Fornax A. Ionized gas from NGC 1310 produces a Faraday screen that depolarizes the background radiation (Fomalont et al. 1989; Schulman \& Fomalont 1992). Although the galaxy is located 20.5 away from the center of Fornax A on the plane of the sky and in the outer region of the primary beam of the VLA, HI emission is well detected. Figures 5 and 6 present the results of the Hi observations in the same form as those for NGC 1317. The approaching gas is located on the eastern side of the galaxy. If the spiral arms are trailing, then the northern part of NGC 1310 is the near side. The Hi distribution is slightly asymmetric and the emission is stronger on the eastern side. We estimate a total mass of atomic hydrogen of $4.8 \times 10^{8} M_{\odot}$ for NGC 1310 , which is about 20 times higher than the upper limit on the $\mathrm{H}_{2}$ mass set by Horellou et al. (1995).

Comparing the extent of the Hi disk to that of the optical galaxy, one finds that the isocontour of column density $10^{20} \mathrm{~cm}^{-2}$ roughly coincides with the blue isophote at the 25 th mag $\operatorname{arcsec}^{-2}$. This is unusual compared to Sc galaxies in the field, which normally have an Hi diameter larger than their $\mathrm{D}_{25}$ diameter by a factor of $1.88 \pm 0.08$ (Cayatte et al. 1994). However, NGC 1310 is not significantly HI deficient compared to the isolated Sc galaxies of Haynes \& Giovanelli (1984) (HI def $=0.17$ in the $\log$ ). The small size of the disk $\left(\sim 2^{\prime}\right.$ across $)$ together with the location of NGC 1310 in the outer part of the primary beam of the VLA make it difficult to say more about its Hi properties.

\subsection{NGC 1316 (Fornax A)}

- The spheroid. Huchtmeier \& Richter (1989) give an upper limit on the HI integrated line intensity of $4.5 \mathrm{Jy} \mathrm{km} \mathrm{s}{ }^{-1}$, which corresponds to $3.7 \times 10^{8} M_{\odot}$ at our adopted distance. We didn't detect any $\mathrm{HI}$ in the central region of the galaxy down to a $5 \sigma$ limit of $10^{7} M_{\odot}$ per beam and per channel.

If, however, the Hi emission is as broad in velocity as the $\mathrm{CO}$ detected in the central region (see next section) then the limit is $8.5 \times 10^{7} M_{\odot}$. This could be even higher if the $\mathrm{HI}$ is extended over several beams. Very extended Hi , moreover, will be missed in the present observations because the quality of the data at the shortest spacings is limited by the presence of $34 \mathrm{Jy}$ of continuum emission. A safe limit to the HI emission in the main body of NGC 1316 is $10^{8} M_{\odot}$.

We did not detect Hi absorption towards the nuclear continuum source which has a flux density of $220 \mathrm{mJy}$ at $1412 \mathrm{MHz}$. The $5 \sigma$ column density limit is $1 \times$ $10^{18} T_{\mathrm{s}} \mathrm{cm}^{-2}$, where $T_{\mathrm{s}}$ is the spin temperature (in $\mathrm{K}$ ) of the absorbing atomic hydrogen.

The four Hidetections in the outer regions of NGC 1316 are all far outside the main body of NGC 1316 and lie at or close to the edge of the faint optical shells and X-ray emission of NGC 1316. Each clump contains 1 to $2 \times 10^{7} M_{\odot}$ of $\mathrm{HI}$. The southern clumps are associated with optical emission line gas: the HII region SH2 and the EELR.

- SH2. This H II region has a size of $11^{\prime \prime} 5 \times 33^{\prime \prime} 5(135 \mathrm{pc} \times$ $316 \mathrm{pc}$ ); it is located 6!7, or $36.2 \mathrm{kpc}$ south of the nucleus and has a radial velocity of $-105 \pm 8 \mathrm{~km} \mathrm{~s}^{-1}$ relative to it 

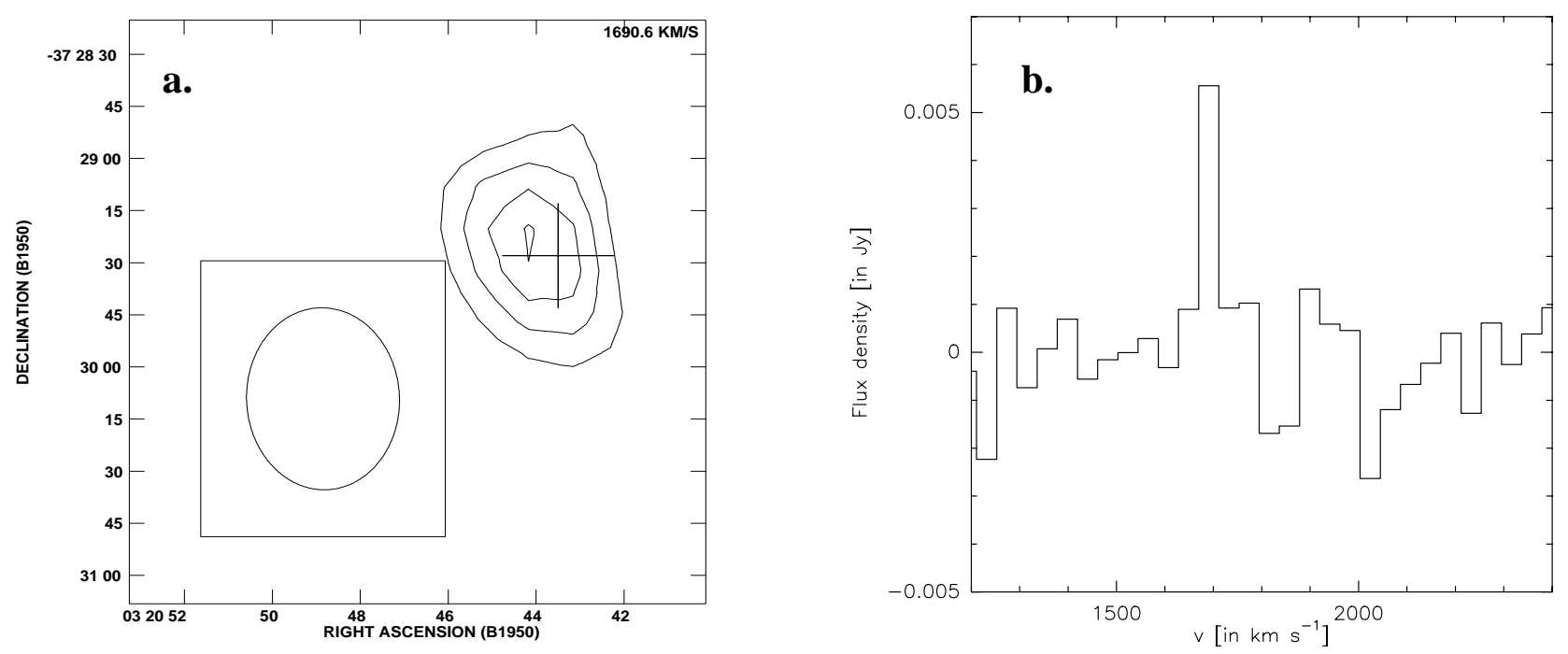

Fig. 7. a) Contour map of the integrated Hi line emission in the Hit region SH2. The levels are 2, 3, 4 and 5 mJy/beam. The first contour corresponds to the $2 \sigma$ level. The circle shows the size of the beam. The cross marks the optical position. b) Integrated Hi spectrum of the HiI region $\mathrm{SH} 2$.

(S80). S80 notes that a "blue fuzz", probably an associated complex of OB stars, is visible near the HII region.

The HI emission in SH2 appears in one channel centered at $v=1690 \mathrm{~km} \mathrm{~s}^{-1}$ (our channel width is $41.7 \mathrm{~km} \mathrm{~s}^{-1}$ ) (see Fig. 7). This is in agreement with the velocity of the ionized gas quoted by Schweizer. The HI mass is about $2 \times 10^{7} M_{\odot}$. The distribution is unresolved at our resolution of $52^{\prime \prime} \times 41^{\prime \prime}(4.7 \times 3.7 \mathrm{kpc})$.

- The EELR. This extended emission line region is located $5 ! 3$, or $28.6 \mathrm{kpc}$ from the nucleus and has a size of $81^{\prime \prime} \times 27^{\prime \prime}$, or $7.3 \mathrm{kpc} \times 2.4 \mathrm{kpc}$ (for our distance of $18.6 \mathrm{Mpc}$ ). On the plane of the sky, it appears on a region of high surface brightness in the loop called $L_{1}$ by S80 and near a major dust lane.

The HI associated with the EELR is $\sim 1 \times 10^{7} M_{\odot}$ and also unresolved by our beam.

The two northern clumps are located at the outer edge of a plateau in the optical surface brightness distribution (Fig. 2, see also Fig. 7 of S80). The velocities of the Hi clumps are (from north to south through west) 1857 , 2000, 1750 (EELR) and 1690 (SH2), respectively, following the general rotation pattern on the central CO (next section) and suggesting that the clumps may be the leftovers of a more widespread Hi structure, possibly a tidal shred. The location and velocity structure of the HI are reminiscent of other shell galaxies such as Centaurus A (Schiminovich et al. 1994) and NGC 2865 (Schiminovich et al. 1995).

\section{Molecular gas}

\subsection{The central region of NGC 1316 (Fornax A)}

Results from the CO observations are presented in Figs. 8 to 10 and the line parameters are given in Table 3 . The observations were done by two observers in two separate sessions, and the observing strategy was slightly different.
During the first observing session (see top panel of Fig. 8), we mapped the $\mathrm{CO}$ emission along the axis with a position angle $\mathrm{PA}=142^{\circ}$, given by $\mathrm{S} 80$ as the direction of the maximum extent of the ionized gas and probably the rotation axis of the spheroid (perpendicular to the optical major axis). During the second session (Fig. 8, bottom panel), the grid was in RA/DEC. Emission is detected north-west and south-east of the nucleus as well as toward the center. The intensities in the NW region are about twice as strong as in the $\mathrm{SE}$, both in $\mathrm{CO}(1-0)$ and $\mathrm{CO}(2-1)$. The $\mathrm{CO}$ distribution roughly follows that of the dust along the minor axis and to the NE.

- Emission was detected toward the central position. The averaged central spectra obtained during both observing sessions are shown in Fig. 9. The noise level in our $\mathrm{CO}(1-0)$ spectrum is about 5 times lower than that in the spectrum obtained by Wiklind \& Henkel (1989) and the bandwidth is larger, which makes it easier to see the line structure. The integrated line intensity we infer is about three times lower than that reported in that work, but it may agree within the not quoted error bars of Wiklind \& Henkel. Sage \& Galletta (1993) had detected CO(1-0) at two positions $30^{\prime \prime}$ to the $\mathrm{SE}$ and $50^{\prime \prime}$ to the $\mathrm{NW}$ of the nucleus but not toward the center. The fluxes and the limits quoted agree with ours within the uncertainties. The $\mathrm{CO}(1-0)$ line seems to be very broad $\left(\sim 500 \mathrm{~km} \mathrm{~s}^{-1}\right.$ at the base) and to consist of three components. It is likely that the molecular gas distribution is discrete and that the low-velocity component arises from emission from the SE that enters the $43^{\prime \prime}$ beam, whereas the hint of emission at high velocity $\left(\sim 1900 \mathrm{~km} \mathrm{~s}^{-1}\right)$ comes from the NW concentration. All those features are, if present at all, much weaker in the $\mathrm{CO}(2-1)$ spectrum taken with a narrower beam. We calculate a mass of the $\mathrm{H}_{2}$ gas of $\sim 10^{8} M_{\odot}$ within the inner $43^{\prime \prime}(3.9 \mathrm{kpc})$. This estimate of mass is based upon a standard Galactic conversion factor. 


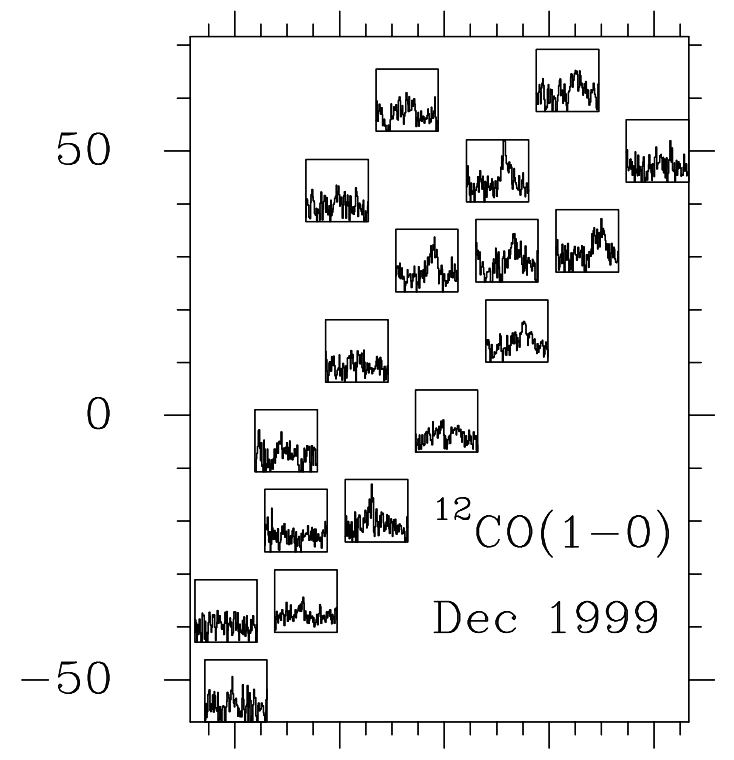

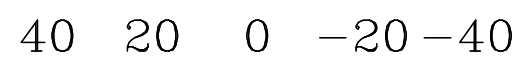

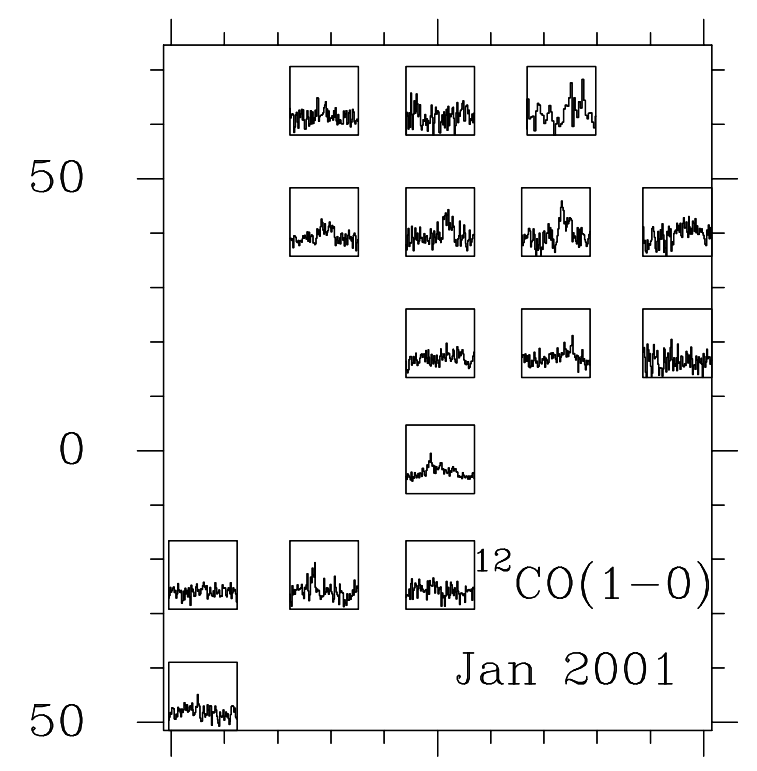

50

0

$-50$

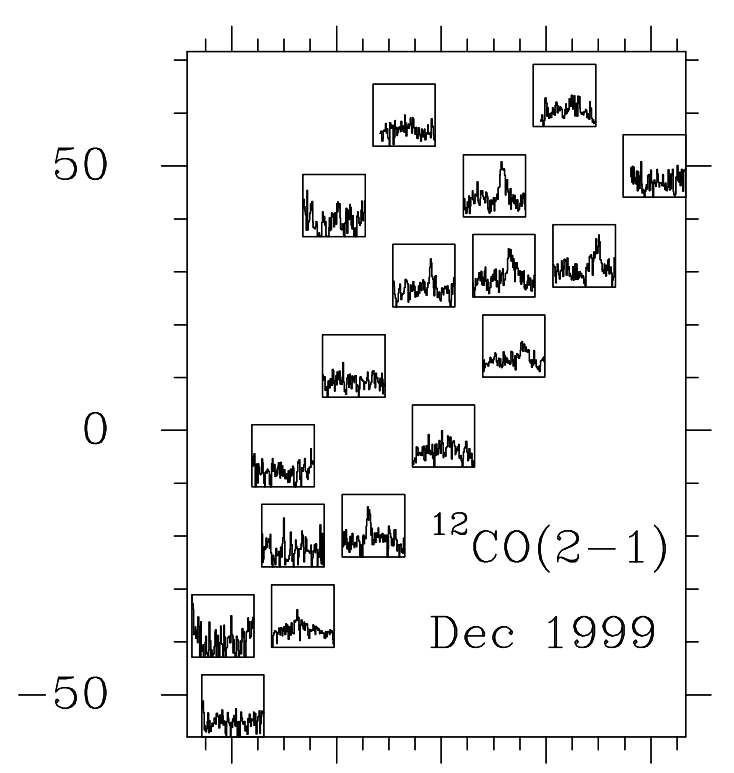

$40 \quad 20 \quad 0 \quad-20-40$

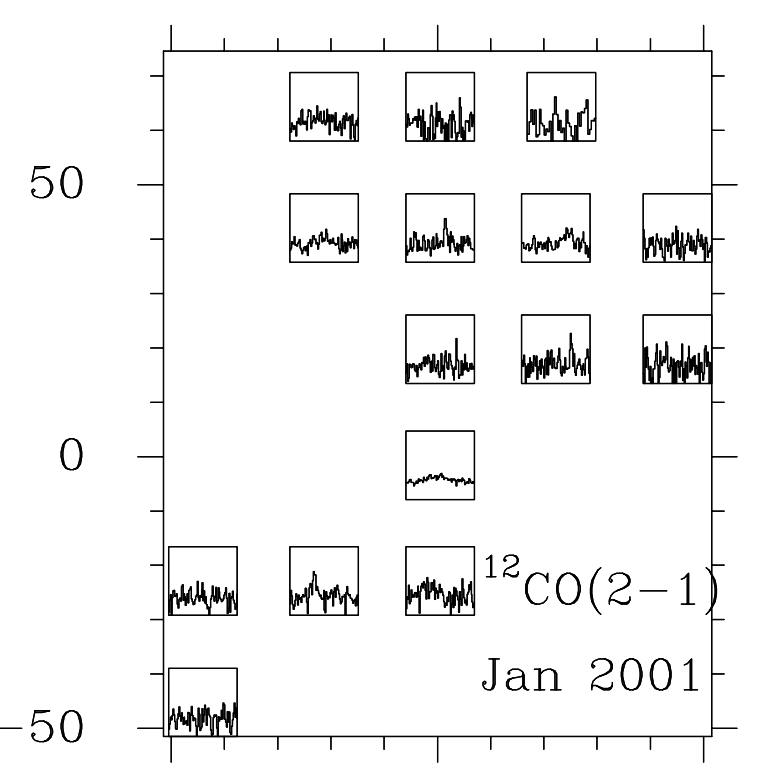

50

0

Fig. 8. Top: $\mathrm{CO}(1-0)$ and $\mathrm{CO}(2-1)$ spectra obtained in December 1999. The offsets are in arcseconds. The $x$ scale ranges from 1000 to $2300 \mathrm{~km} \mathrm{~s}^{-1}$. The $y$ scale (main-beam temperature, $T_{\mathrm{mb}}$ ) ranges from -0.01 to $0.03 \mathrm{~K}$. The velocity resolution of the $\mathrm{CO}(1-0)$ spectra is $14.5 \mathrm{~km} \mathrm{~s}^{-1}$, that of the $\mathrm{CO}(2-1)$ spectra is $14.6 \mathrm{~km} \mathrm{~s}^{-1}$. Bottom: results of the January 2001 observing sesion. The $x$ scale ranges from 1100 to $2300 \mathrm{~km} \mathrm{~s}^{-1}$. The $y$ scale and the velocity resolution are the same as for the spectra above.

- The $N W$ concentration is centered at $\Delta \alpha=-14^{\prime \prime}$, $\Delta \delta=+37^{\prime \prime}$ relative to the central position, that is, at a projected distance of $3.5 \mathrm{kpc}$ from the nucleus. The emission is extended on the scale of the $22^{\prime \prime} \mathrm{CO}(2-1)$ beam, and the measured apparent diameter at half maximum intensity is $35^{\prime \prime}$, which correspond to a true extent of $\theta_{\text {source }}=27^{\prime \prime}$, or a linear size of $\sim 2.5 \mathrm{kpc}$. This is consistent with the $\mathrm{CO}(1-0)$ measurements, which yield an apparent diameter of the half peak intensity of $50^{\prime \prime}$ (and a true source size of $24^{\prime \prime}$ ). Velocities around $1850 \mathrm{~km} \mathrm{~s}^{-1}$ are found in the NW region, that is, $\sim 70 \mathrm{~km} \mathrm{~s}^{-1}$ higher than the systemic velocity of NGC 1316. A slight east-west velocity gradient is observed, with higher velocities to the west.

From the apparent extent of the northwest $\mathrm{CO}$ concentration measured above and the $\mathrm{CO}(1-0)$ peak intensity, we derive a mass of molecular hydrogen within that region of $2.2 \times 10^{8} M_{\odot}$. 
Table 3. Line parameters.

\begin{tabular}{|c|c|c|c|c|c|c|c|c|c|c|c|}
\hline & & & & ${ }^{12} \mathrm{CO}(1-0)$ & & & & ${ }^{12} \mathrm{CO}(2-1)$ & & & \\
\hline$X$ & $Y$ & $\mathrm{RA}$ & DEC & $v$ & & $\int T \mathrm{~d} v$ & $\sigma$ & $v$ & $\Delta v$ & $\int T \mathrm{~d} v$ & $\sigma$ \\
\hline "1 & " & $" 1$ & & $\mathrm{~km} \mathrm{~s}^{-1}$ & $\mathrm{~km} \mathrm{~s}^{-1}$ & $\mathrm{~K} \mathrm{~km} \mathrm{~s}^{-1}$ & $\mathrm{mK}$ & $\mathrm{km} \mathrm{s}^{-1}$ & $\mathrm{~km} \mathrm{~s}^{-1}$ & $\mathrm{~K} \mathrm{~km} \mathrm{~s}^{-1}$ & $\mathrm{mK}$ \\
\hline (1) & $(2)$ & $(3)$ & (4) & $(5)$ & (6) & (7) & (8) & (9) & & & \\
\hline & & & & & & 1999 observing & session & & & & \\
\hline 0 & +22 & +13.5 & -17.3 & $1531 \pm 13$ & $129 \pm 58$ & $2.0 \pm 0.6$ & 5.9 & $1550 \pm 6$ & $88 \pm 13$ & $1.9 \pm 0.3$ & 4.9 \\
\hline 0 & +44 & +27.1 & -34.7 & $1557 \pm 14$ & $128 \pm 27$ & $1.1 \pm 0.2$ & 3.5 & $1525 \pm 7$ & $75 \pm 17$ & $0.9 \pm 0.2$ & 3.0 \\
\hline-22 & -44 & -9.8 & +48.2 & $1809 \pm 8$ & $167 \pm 20$ & $4.3 \pm 0.4$ & 5.0 & $1818 \pm 6$ & $136 \pm 14$ & $3.3 \pm 0.3$ & 4.4 \\
\hline 0 & -44 & -27.1 & +34.7 & $1914 \pm 16$ & $263 \pm 31$ & $4.5 \pm 0.5$ & 5.6 & $1919 \pm 11$ & $178 \pm 31$ & $3.0 \pm 0.4$ & 4.7 \\
\hline 0 & 0 & 0 & 0 & & & $<2.4$ & 5.1 & & & $<2.4$ & 5.0 \\
\hline 0 & -22 & -13.5 & +17.3 & $1813 \pm 16$ & $268 \pm 42$ & $3.4 \pm 0.4$ & 4.4 & $1859 \pm 11$ & $186 \pm 24$ & $2.0 \pm 0.2$ & 3.0 \\
\hline-22 & -22 & +3.8 & +30.9 & $1778 \pm 9$ & $178 \pm 21$ & $3.5 \pm 0.4$ & 4.8 & $1797 \pm 5$ & $67 \pm 14$ & $1.4 \pm 0.2$ & 4.3 \\
\hline-22 & 0 & +17.3 & +13.5 & & & $<2.4$ & 5.1 & & & $<2.1$ & 4.2 \\
\hline-22 & -66 & -23.3 & +65.6 & $1835 \pm 17$ & $224 \pm 38$ & $3.4 \pm 0.5$ & 5.8 & & & $<2.1$ & 4.7 \\
\hline-22 & +22 & +30.9 & -3.8 & & & $<3.3$ & 7.1 & & & $<2.4$ & 5.4 \\
\hline-44 & -44 & +7.6 & +61.8 & & & $<2.7$ & 5.9 & & & $<2.1$ & 4.2 \\
\hline 0 & -66 & -40.6 & +52 & & & $<2.7$ & 5.9 & & & $<2.4$ & 5.4 \\
\hline-44 & -22 & +21.1 & +44.4 & & & $<2.7$ & 6.1 & & & $<3.6$ & 7.6 \\
\hline 0 & +66 & +40.6 & -52 & & & $<3.3$ & 7.3 & & & $<2.4$ & 5.0 \\
\hline-11 & -33 & -11.6 & +32.8 & $1807 \pm 19$ & $206 \pm 48$ & $3.5 \pm 0.7$ & 7.7 & $1802 \pm 11$ & $202 \pm 32$ & $3.5 \pm 0.4$ & 4.6 \\
\hline-11 & +55 & +42.5 & -36.6 & & & $<2.7$ & 5.9 & & & $<4.8$ & 10.6 \\
\hline-11 & +33 & +29.0 & -19.2 & & & $<2.4$ & 5.0 & & & $<3.3$ & 7.0 \\
\hline & & & & & & 2001 observing & session & & & & \\
\hline 0.0 & 0.0 & 0 & 0 & 1634 & 343 & 3.4 & 2.1 & 1653 & 208 & 2.0 & 2.1 \\
\hline-27.7 & -35.5 & 0 & +45 & $1829 \pm 9$ & $157 \pm 22$ & $3.2 \pm 0.4$ & 5.4 & $1795 \pm 4$ & $48 \pm 9$ & $1.7 \pm 0.3$ & 3.4 \\
\hline-10.4 & -49.0 & -22 & +45 & $1846 \pm 12$ & $190 \pm 25$ & $4.3 \pm 0.5$ & 6.4 & $1910 \pm 13$ & $186 \pm 33$ & $2.9 \pm 0.4$ & 5.4 \\
\hline+7.8 & -63.2 & -45 & +45 & $1900 \pm 35$ & $400 \pm 70$ & $4.7 \pm 0.8$ & 4.6 & & & $<1.5$ & 9 \\
\hline-45.0 & -21.9 & +22 & +45 & $1737 \pm 14$ & $242 \pm 27$ & $2.6 \pm 0.3$ & 3.4 & $1697 \pm 27$ & $270 \pm 53$ & $2.6 \pm 0.3$ & 4.8 \\
\hline-59.2 & -40.0 & +22 & +68 & $1671 \pm 30$ & $207 \pm 53$ & $1.4 \pm 0.4$ & 4.8 & $1578 \pm 52$ & $394 \pm 96$ & $3.3 \pm 0.9$ & 6.8 \\
\hline+3.8 & -30.9 & -22 & +22 & $1898 \pm 22$ & $292 \pm 60$ & $2.7 \pm 0.4$ & 4 & $1967 \pm 5$ & $41 \pm 10$ & $1.6 \pm 0.4$ & 9 \\
\hline-3.8 & +30.9 & +22 & -22 & $1514 \pm 6$ & $73 \pm 13$ & $1.5 \pm 0.3$ & 5.3 & $1529 \pm 7$ & $103 \pm 19$ & $2.8 \pm 0.4$ & 7 \\
\hline-13.5 & -17.3 & 0 & +22 & $1805 \pm 41$ & $333 \pm 75$ & $1.1 \pm 0.4$ & 4.2 & & & $<1.8$ & 7.8 \\
\hline+21.9 & -45.0 & -45 & +22 & & & $<1.8$ & 7.1 & & & $<4.5$ & 12. \\
\hline+13.5 & +17.3 & 0 & -22 & $1506 \pm 21$ & $142 \pm 44$ & $1.1 \pm 0.3$ & 4.7 & $1500 \pm 36$ & $327 \pm 74$ & $2.7 \pm 1.0$ & 10. \\
\hline-21.9 & +45.0 & +45 & -22 & & & $<0.9$ & 4. & & & $<2.1$ & 8. \\
\hline-7.8 & +63.2 & +45 & -45 & & & $<1.2$ & 4.4 & & & $<2.4$ & 9. \\
\hline-41.9 & -53.6 & 0 & +68 & & & $<1.8$ & 5.4 & & & $<4.5$ & 12. \\
\hline-23.7 & -67.7 & -23 & +68 & $1963 \pm 29$ & $334 \pm 47$ & $3.6 \pm 0.8$ & 8.7 & $1960 \pm 32$ & $72 \pm 56$ & $1.2 \pm 1.0$ & 15. \\
\hline & & & & Combined & data and & outer HiI regions & & & & & \\
\hline 0.0 & 0.0 & 0 & 0 & 1559 & 189 & 2.4 & 2.1 & 1642 & 213 & 1.9 & 2.1 \\
\hline EELR & & & & & & & 3.2 & & & & 3.8 \\
\hline $\mathrm{SH} 2$ & & & & & & & 3.1 & & & & 4.1 \\
\hline
\end{tabular}

(1), (2): Offset positions after rotation of the coordinate system by $\mathrm{PA}=142^{\circ}$.

(3), (4): Offset positions in RA and DEC.

(5) to (7): For all positions but the center we give the parameters of the Gaussian lines fitted to the $\mathrm{CO}(1-0)$ spectra: central velocity, linewidth, integrated main-beam intensity. For the center position where the lines are clearly not Gaussian, we give the velocity of the centroid, the linewidth and the main-beam intensity integrated over the velocity range of the line (see Fig. 9). For non-detections, the limit on the integrated intensity was taken as $3 \times \sigma \times 150 \mathrm{~km} \mathrm{~s}^{-1}$.

(8): Noise per $14.5 \mathrm{~km} \mathrm{~s}^{-1}$ channel.

(9) to (12): Same as Cols. 5 to 8 but for the $\mathrm{CO}(2-1)$ spectra.

- In the southeast, the strongest emission is detected $22^{\prime \prime}(\sim 2 \mathrm{kpc})$ away from the nucleus and is half as strong at $44^{\prime \prime}$. It corresponds to a $\mathrm{H}_{2}$ mass of $0.86 \times 10^{8} M_{\odot}$. The velocities measured there are of the order of $1550 \mathrm{~km} \mathrm{~s}^{-1}$, that is, $\sim 230 \mathrm{~km} \mathrm{~s}^{-1}$ lower than the systemic velocity of NGC 1316.
- The CO velocity fields are displayed in Figs. 10c and 10d. They are fairly regular. The systemic velocity of NGC 1316, however, is likely to be higher than suggested from those interpolated contour maps. As discussed above for the central position, the low velocity wing around $1500 \mathrm{~km} \mathrm{~s}^{-1}$ could be due to emission from the NW 


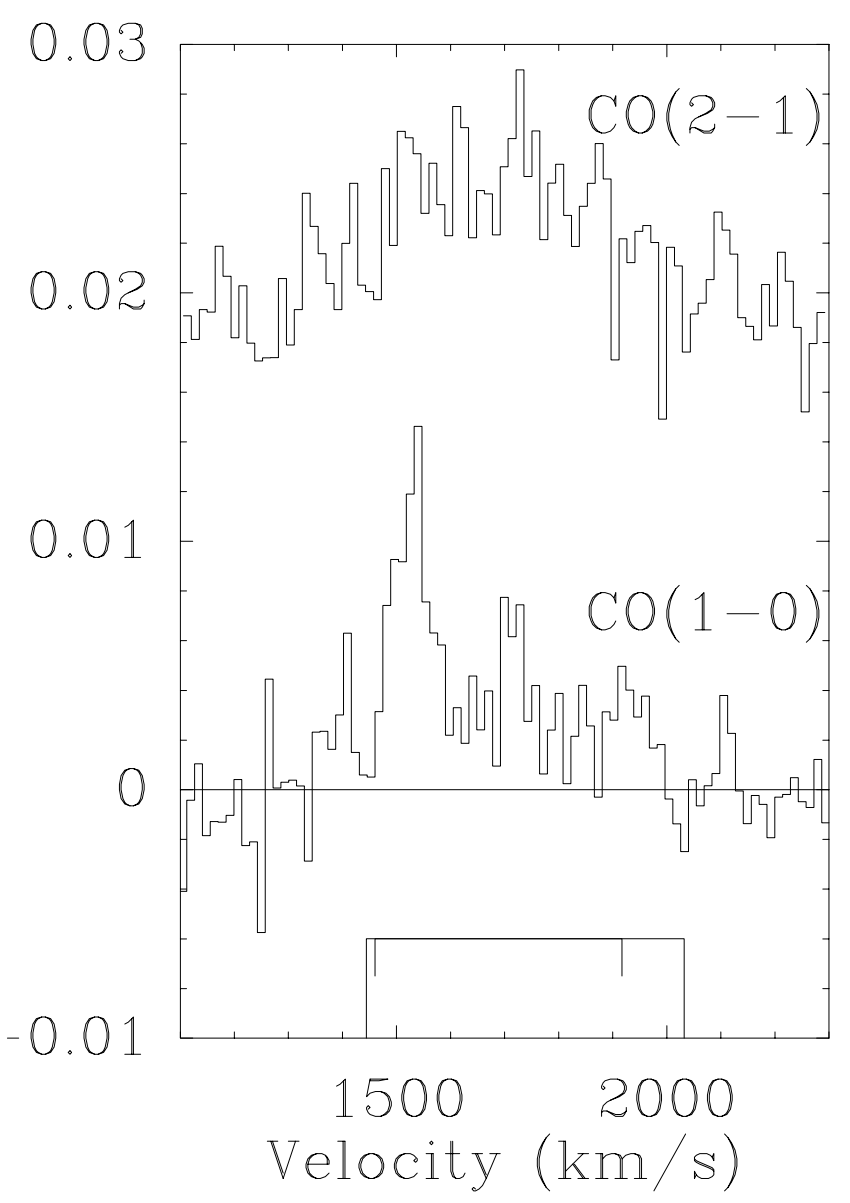

Fig. 9. $\mathrm{CO}(1-0)$ detection toward the center of NGC 1316. The line is broad and seems to consist of three components. The $y$ axis shows the main-beam temperature in Kelvin. The width of the window set to subtract a baseline is shown on the $x$ axis: 1444 to $2032 \mathrm{~km} \mathrm{~s}^{-1}$ for the $\mathrm{CO}(1-0)$ spectrum as indicated by the box, and 1460 to $1917 \mathrm{~km} \mathrm{~s}^{-1}$ for the $\mathrm{CO}(2-1)$ spectrum as indicated by the ticks. The $\mathrm{CO}(2-1)$ line seems to be narrower than the $\mathrm{CO}(1-0)$ one, which is to be expected if the molecular gas is located in the dusty regions offset from the center and located outside the narrower $\mathrm{CO}(2-1)$ beam. The spectra have been produced by averaging the data taken during both observing sessions.

concentration that enters the beam. The centroid velocity of $1559 \mathrm{~km} \mathrm{~s}^{-1}$ given in Table 2 for the central position would therefore be too low and affect the interpolation in the contour map.

\subsection{The outer emission-line regions}

We have searched for $\mathrm{CO}$ emission toward the giant HII region $\mathrm{SH} 2$ and the extended emission line region but have not detected any, down to the noise levels quoted in Table 3. For SH2 as for EELR, this corresponds to a $5 \sigma$ limit on the $\mathrm{H}_{2}$ mass of $\sim 10^{7} M_{\odot}$ per $43^{\prime \prime}$ beam per $14.5 \mathrm{~km} \mathrm{~s}^{-1}$ channel.

\section{Discussion}

While $\mathrm{CO}$ is clearly detected in the central region of NGC 1316, atomic hydrogen is not. The distribution of the molecular gas follows that of the dust, and its kinematics provides new insight into the dynamical history of the system. HI is detected in the outer part where no $\mathrm{CO}$ is found down to a similar mass limit.

\subsection{Comparison with other components of the ISM}

- The central part of NGC 1316

The dust patches are very visible in the $B-I H S T$ image of NGC 1316 displayed in Fig. 11c. They appear on the NW and the SE side of the nucleus, roughly aligned along the minor axis of the galaxy. The SE lane can be traced to within 1 arcsec from the center, which led Schweizer (1980) to suggest that it lies on the near side of the galaxy. The molecular gas distribution at $22^{\prime \prime}$ resolution follows roughly the distribution of the dust.

Ionized gas was found by $\mathrm{S} 80$ to be associated with the dust and distributed in a rotating disk with extremal velocities $\pm 350 \mathrm{kms}^{-1}$ at $84^{\prime \prime} \mathrm{NW}$ and $35^{\prime \prime} \mathrm{SE}$. The SE side of the disk is approaching. The extreme range of $\mathrm{CO}$ velocities that we measure on either side of the nucleus, 1500 to $1960 \mathrm{~km} \mathrm{~s}^{-1}$ at $32^{\prime \prime} \mathrm{SE}$ and $72^{\prime \prime} \mathrm{NW}$, is thus smaller than the range of velocities in the ionized disk.

Shaya et al. (1996) used HST $V$ and $I$ band images of the central region (a field of view of $70^{\prime \prime}$ ) to analyze the three-dimensional distribution of the dust. They derived a small amount of extinction toward the very center, $A_{V}<0.4 \mathrm{mag}$ and $A_{I}<0.2 \mathrm{mag}$, and $A_{V} \sim 1.5$ in the regions of maximum extinction. The amount of molecular gas that we infer from the $\mathrm{CO}$ observations correlates with the amount of extinction: we detect only $\sim 10^{8} M_{\odot}$ in the center and about twice as much in the dusty NW region.

The radio jet observed by Geldzahler \& Fomalont (1984) could be deflected by interstellar gas, especially in the NW. Figure 11a shows a superposition of our $\mathrm{CO}(2-1)$ map and the radio jet at $\lambda 20 \mathrm{~cm}$. The direction of the central jet changes abruptly in the NW just below the peak in the $\mathrm{CO}$ distribution. There is a hint of a similar bending near the SE peak in the CO.

The most luminous galaxies in the far-infrared are mergers. NGC 1316 was detected by IRAS in the four bands but its far-infrared luminosity is moderate $(\sim 2 \times$ $\left.10^{9} L_{\odot}\right)$. The $L_{\text {FIR }}$ to $M\left(\mathrm{H}_{2}\right)$ ratio, often used as an indicator of the efficiency at which molecular gas is converted into new stars, is $\sim 5$. This is lower than the average value of $\sim 15$ that we calculated for the sample of ellipticals observed by Wiklind et al. (1995) (after exclusion of the two galaxies with the highest values of $L_{\mathrm{FIR}} / M\left(\mathrm{H}_{2}\right)$ ratios, an S0 and a galaxy larger than the beam size, for which the $\mathrm{H}_{2}$ mass must have been underestimated). Nevertheless, this may not mean that the star formation efficiency of NGC 1316 is lower than in other ellipticals since it is known that some of the far-infrared emission is not related to massive ionizing stars, but to dust heated by the 

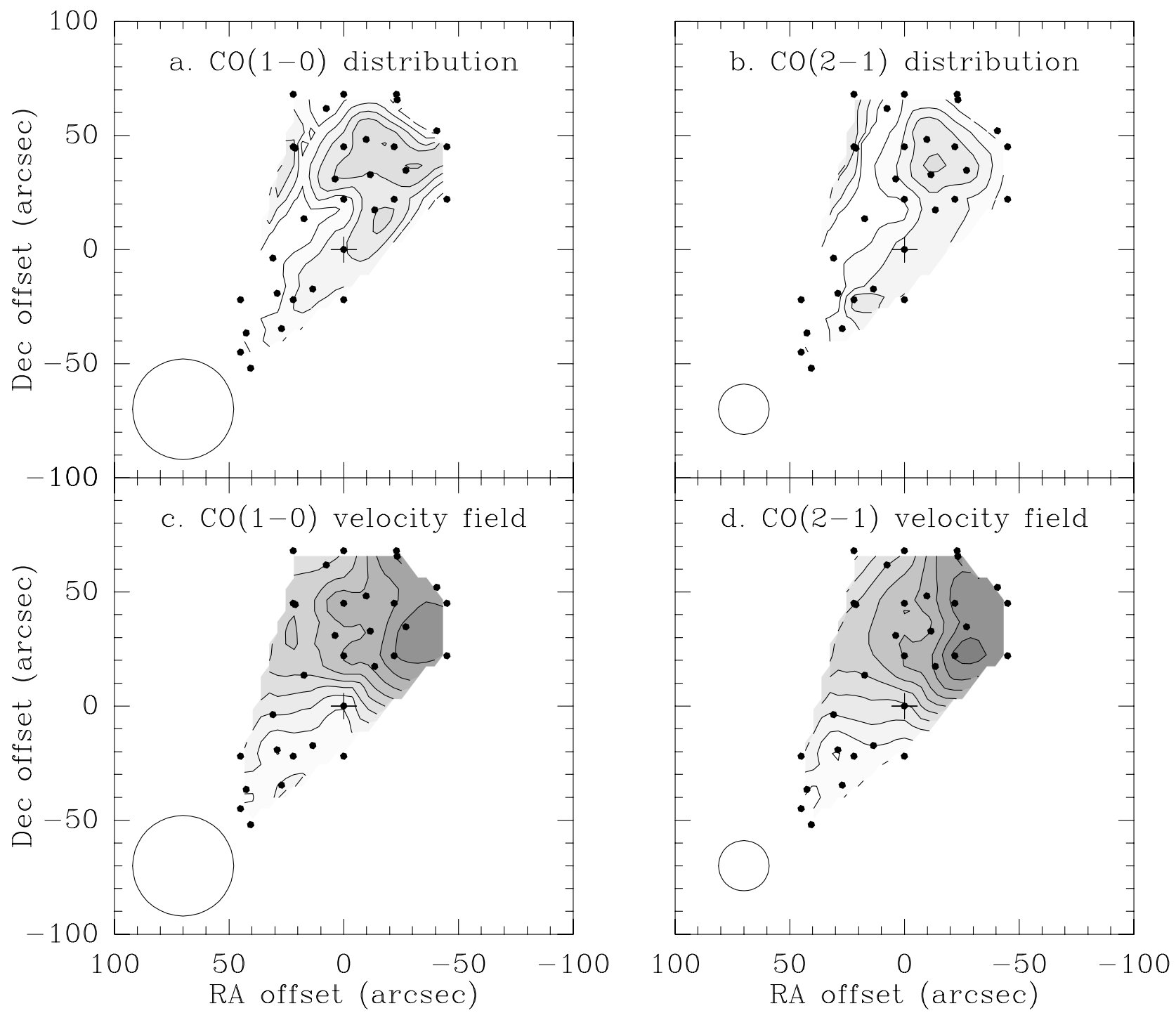

Fig. 10. a) and c) Contour map of the $\mathrm{CO}(1-0)$ distribution and corresponding velocity field. The levels of the total intensity map go from 0.5 to $6 \mathrm{~K} \mathrm{~km} \mathrm{~s}^{-1}$ in steps of $1 \mathrm{~K} \mathrm{~km} \mathrm{~s}^{-1}$. The levels of the velocity map go from 1500 to $2000 \mathrm{~km} \mathrm{~s}^{-1}$ in steps of $50 \mathrm{~km} \mathrm{~s}^{-1}$. The circle shows the size of the beam. The black dots indicate the observed positions. The central position is marked by a cross. b) and d) Contour map of the $\mathrm{CO}(2-1)$ distribution and corresponding velocity field. The levels are the same as for the $\mathrm{CO}(1-0)$.

overall stellar radiation field. Moreover, the Galactic COto- $\mathrm{H}_{2}$ conversion factor may not apply to ellipticals or it may vary from one elliptical to another. For example, an elliptical galaxy that has accreted a gas-rich but metalpoor companion could be $\mathrm{CO}$-poor and its $\mathrm{H}_{2}$ mass would be underestimated.

\section{- The outer HII regions}

It is interesting that $\mathrm{SH} 2$, despite being so much smaller than EELR, possesses about twice as much atomic gas. Mackie \& Fabbiano (1998) give an $\mathrm{H} \alpha$ luminosity $L_{\mathrm{H} \alpha}=5.46 \times 10^{5} L_{\odot}$ for the EELR, and a mass of ionized gas $M_{\mathrm{HII}}=5 \times 10^{3} M_{\odot}$ (when corrected for our adopted distance). If the EELR were photoionized by hot stars, this quantity of ionized gas could be maintained by $\sim 40$ O-type stars. However, Mackie and Fabbiano argued that there is no evidence of a population of young stars associated with the EELR and suggested that the ionized gas is produced by shocks. Although Mackie \& Fabbiano do not quote an ionized gas mass for SH2, it appears from their $\mathrm{H} \alpha$ image, which has been smoothed to a resolution of $4 . .3$, that the $\mathrm{H} \alpha$ flux is of the order of $3 \times 10^{-16}$ ergs s$^{-1} \mathrm{~cm}^{-2}$, integrated over the $1^{\prime \prime} .5 \times 3^{\prime \prime} .5$ size reported by $\mathrm{S} 80$. At our adopted distance, such recombination emission can be maintained by a single O-type star. For comparison, the giant HII region NGC 604 in the Local Group galaxy M 33 contains $3 \times 10^{6} M_{\odot}$ of ionized gas (Churchwell \& Goss 1999) and approximately 200 Otype stars (González Delgado \& Pérez 2000). Although the $\mathrm{H} \alpha$ emission of $\mathrm{SH} 2$ may signify recent star-forming activity, the population of massive stars is not large in comparison with the mass of neutral gas in the vicinity, $M_{\mathrm{H} \text { I }} \approx 2 \times 10^{7} M_{\odot}$. 


\subsection{The absence of $\mathrm{HI}$ emission and absorption in the central spheroid}

In the central region of NGC 1316 the $\mathrm{HI} \lambda 21 \mathrm{~cm}$ line is not clearly detected in emission nor is there any noticeable absorption against the continuum emission. Our limit on HI emission in the spheroid of NGC 1316 could represent a mass of $\mathrm{H}$ as high as $10^{8} M_{\odot}$. The limit on absorption against the central continuum source corresponds to a column density of $\mathrm{H}<1 \times 10^{18} T_{\mathrm{s}} \mathrm{cm}^{-2}$, implying that only gas with spin temperature $T_{\mathrm{s}}<100 \mathrm{~K}$ could reasonably have been detected. The upper limit on Hi emission may at first sight seem surprising in view of the large amounts of dust and molecular gas in the spheroid. Fornax A is not unique in this respect: other well known elliptical galaxies and merger remnants show $\mathrm{CO}$ and dust but no detectable $\mathrm{H}$ in their centers (e.g. NGC 7252, Wang et al. 1992; Hibbard et al. 1994). In contrast there are smaller elliptical galaxies or merger remnants that have dust, $\mathrm{CO}$ and plenty of $\mathrm{H}$ all the way in to their centers. Examples are NGC 5128, NGC 3656, and Arp 230, all of which have disks that are viewed nearly edge-on (Schiminovich et al. 1994; Eckart et al. 1990; Balcells et al. 2001; Sofue et al. 1993; Schiminovich et al. 2001; Galletta et al. 1997). The $\mathrm{H}$-deficient and $\mathrm{H}$-rich systems also differ in the size of the spheroid and presence of extended X-ray emission. However, the detectability of Hi absorption may also depend on whether a gaseous disk is present and if so how it is inclined to the observer's line of sight.

The CO emission from NGC 1316 probably arises in the surfaces of molecular clouds. Any neutral atomic gas associated with such molecular clouds may cover too little of the central continuum source to be detectable in absorption in the $\lambda 21 \mathrm{~cm}$ line at the angular resolution and sensitivity of the available measurements. The high pressure of the hot X-ray-emitting gas and the shocks associated with the merger events may help confine the neutral gas in small clouds, while the X-radiation keeps the more dilute gas ionized.

Finally, it is interesting to consider briefly how the spin temperature of $\mathrm{H}$ in the central region of NGC 1316 might be affected by the extreme environment there. There are radiative processes that can boost the spin temperature of low-density $\mathrm{H}$ : absorption and stimulated emission induced by the continuum radiation in the $21 \mathrm{~cm}$ line itself (cf. Bahcall \& Ekers 1969), and absorption and fluorescence in the Hi $\mathrm{L} \alpha$ line (Deguchi \& Watson 1985). These processes will not affect the interpretation of optically thin Hi emission lines, but they could make absorption even less readily detectable. Although Fornax A is a bright continuum source at $\lambda 21 \mathrm{~cm}$, the integrated flux density of 0.24 Jy over the central $10^{\prime \prime} \times 30^{\prime \prime}$ (Ekers et al. 1983; Geldzahler \& Fomalont 1984) corresponds to an average continuum brightness temperature of $565 \mathrm{~K}$ and yields an absorption rate $F=0 \rightarrow 1$ of $\rho \approx 7 \times 10^{-11} \mathrm{~s}^{-1}$. The brightness temperature in the extended central jets is lower, $\sim 150 \mathrm{~K}$, which might be a lower limit on the spin temperature of dilute gas in the center. Pumping in the
HI $\mathrm{L} \alpha$ line will have a larger effect on the spin temperature of HI. The average surface brightness of the HI L $\alpha$ line can be estimated from the observed surface brightness in the $\mathrm{H} \alpha$ line, $I \sim 4 \times 10^{-17} \mathrm{erg} \mathrm{s}^{-1} \mathrm{~cm}^{-2} \operatorname{arcsec}^{-2}$ (Kim et al. 1998), with the assumption that a fraction $\eta$ of the Balmer- $\alpha$ emissions are followed by emission in $\mathrm{L} \alpha$. The corresponding pumping rate $F=0 \rightarrow 1$ is $\sim 10^{-7} \eta \mathrm{s}^{-1}$, averaged over the central arcmin of the galaxy. Since $\eta$ is likely to be of the order of unity, this excitation rate is much higher than the mean rate of continuum pumping in the $21 \mathrm{~cm}$ line and is high enough to dominate over spin-changing collisions if the density of the $\mathrm{HI}$-emitting gas is $\$ 10^{2} \mathrm{~cm}^{-3}$. When $\mathrm{L} \alpha$ pumping controls the spin temperature, this temperature is probably $\sim 10^{4} \mathrm{~K}$ (Deguchi \& Watson 1985). Thus the L $\alpha$ pumping could make the $\mathrm{HI}$ line undetectable in absorption in the center of NGC 1316 by ensuring that its spin temperature remains high even where the kinetic temperature might be much lower than $10^{4} \mathrm{~K}$. The true magnitude of the $\mathrm{L} \alpha$ pumping effect is uncertain, of course, because the distributions of dilute neutral gas and of $\mathrm{L} \alpha$ photons are not known.

There is a large amount of hot, ionized, X-ray-emitting gas in the central kpc of NGC 1316, which arises at the expense of neutral gas that might otherwise exist there. In the future, it will be valuable to obtain Hi observations of greater sensitivity and higher resolution to try to trace the neutral gas and its interactions with its environment in more detail.

\subsection{Timescales}

The complex structure of NGC 1316 (five tails or loops of varying morphology) argues for a rich dynamical history and probably a succession of merging events. The presence of shells around ellipticals and minor-axis dusty disks can be interpreted in two different ways: as tracers of the accretion of a small companion onto an already existing elliptical galaxy (e.g. Quinn 1984), or as the returning of tidal material during the merger of two disk galaxies (e.g., Hernquist \& Spergel 1992; Balcells 1997). There is observational evidence that NGC 1316 formed in a major merger $\sim 3$ Gyr ago and has been accreting material more recently, as discussed below.

\subsubsection{A major merger $\sim 3$ Gyr ago}

NGC 1316 has a relaxed morphological appearance within $3^{\prime}$, which indicates that, if the galaxy is the result of a major merger between two disk galaxies, the event must have occurred more than $\sim 2 \times 10^{8}$ years ago.

The population of globular clusters constrains the age of the system. Among 24 star clusters associated with NGC 1316, Goudfrooij et al. (2001) identified four exceptionally luminous ones $\left(M_{V}<12.3\right)$ with near-solar metallicities, whose age of $3.0 \pm 0.5$ Gyr could be determined from comparison with single-burst population 


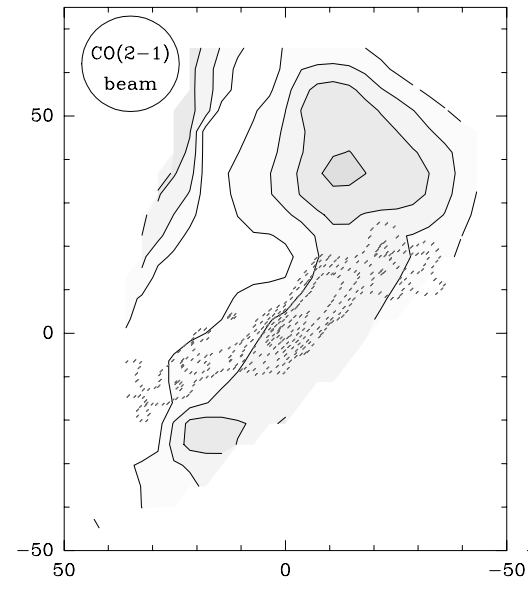

a. $\operatorname{CO}(2-1)$ map

$+20 \mathrm{~cm}$ radio jet

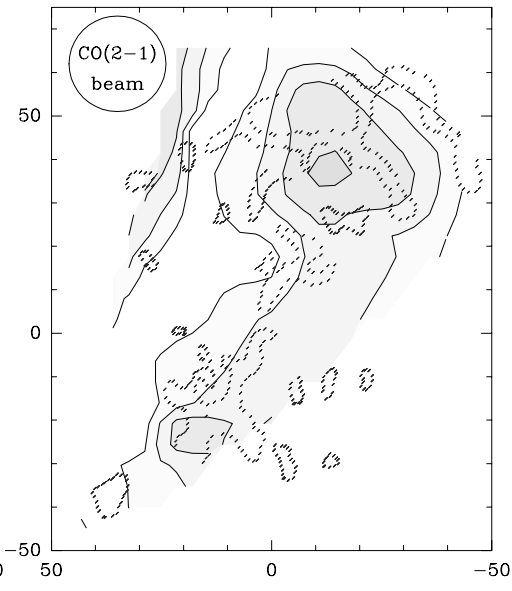

b. $\operatorname{CO}(2-1)$ map

+ sketch of dust lanes

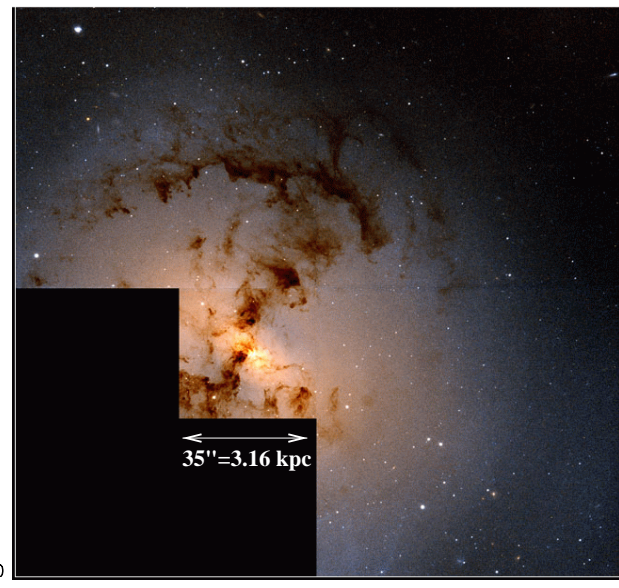

c. HST B-I image

Fig. 11. a) $\mathrm{CO}(2-1)$ contour map overlaid on the $20 \mathrm{~cm}$ radio jet mapped by Geldzahler \& Fomalont (1984). b) $\mathrm{CO}(2-1)$ contour map overlaid on Schweizer (1980)'s sketch of the dust lanes (dots). c) HST B - I image of the central region of NGC 1316 (press release STScI-PR99-06, Grillmair et al. 1999).

models. Those intermediate-age clusters would have formed during a merger of two disk galaxies that resulted in the formation of the elliptical whereas the older ( 14 Gyr old) metal-poor clusters are likely to have belonged to the progenitor disk galaxies. As a remnant of the merger of two disk galaxies, NGC 1316 would be at a more advanced stage than for instance NCC 7252, which is believed to be $0.5-1$ Gyr old and still displays extended tidal tails (Schweizer \& Seitzer 1998).

Optical spectra of the inner $3^{\prime \prime}$ of NGC 1316 have revealed a stellar population of age $\sim 2$ Gyr (Kuntschner 2000). Those stars would therefore have formed from the molecular gas that fell toward the center following the merger event and would have contributed to the depletion of molecular gas observed in the central region today. Strong nuclear $\mathrm{H} \beta$ absorption has been found (Schweizer 1981; Bosma et al. 1985). It might indicate an important component of A-type stars which are part of a poststarburst population.

The X-ray luminosity increases as a result of gas being shock heated or evaporated by electrons in the already existing hot plasma (e.g., Goudfrooij \& Trinchieri 1998) and mass loss from evolving giant stars and supernovae Ia (e.g., Brighenti \& Mathews 1999). Using ROSAT, Kim et al. (1998) detected $\sim 5 \times 10^{8} M_{\odot}($ at $D=18.6 \mathrm{Mpc})$ of hot $\left(\sim 10^{7} \mathrm{~K}\right)$ gas, which is small compared to the amount of hot gas found in some early-type galaxies of comparable optical luminosity. However, Kim et al. (1998) point out that the amount of hot gas is comparable with what would be expected from stellar evolution in $\sim 1$ Gyr. A comparable amount of hot gas is coincident with the tidal tails in the outskirts of NGC 1316 (Mackie \& Fabbiano 1998) and may be due to more recent events.

\subsubsection{More recent infalls}

The presence of loops and ripples as well as of a disk of ionized gas is strong evidence in favor of the infall of a small galaxy onto NGC 1316 less than 1 Gyr ago (Schweizer 1980). In particular, the giant loop called $L_{1}$ by Schweizer is similar to features seen in simulations of shell galaxies (e.g., Quinn 1984; Hernquist \& Quinn 1988) and could be interpreted as due to the infall of a dynamically colder galaxy $\sim 0.5$ Gyr ago. Mackie \& Fabbiano (1998) have argued from the temperature of the hot gas detected in the $L_{1}$ region that $L_{1}$ could be the remnant of a small gas-rich galaxy on a high-velocity encounter $\left(\sim 380 \mathrm{~km} \mathrm{~s}^{-1}\right)$ with NGC 1316, and its origin would therefore be different from that of the other loops which formed in the aftermath of the major, older merger. The extended emission-line region EELR is found in the area of highest surface brightness of $L_{1}$. The fact that we don't detect any $\mathrm{CO}$ from the EELR is not surprising if the infalling galaxy was metalpoor. The low level of detectable Hi emission from that region may be due to the fact that the most of the atomic hydrogen has been stripped, or photo-ionized by the newly formed $\mathrm{OB}$ associations.

The NW concentration could as well be a recently accreted gas-rich galaxy. It has a linear dimension of about $2.5 \mathrm{kpc}$ and contains about $2.2 \times 10^{8} M_{\odot}$ of molecular hydrogen. This $\mathrm{H}_{2}$ mass is comparable to that of NGC 1317, the small ( $15 \mathrm{kpc}$ diameter) spiral companion of NGC 1316 (Horellou et al. 1995). This gas concentration is located $40^{\prime \prime}(3.5 \mathrm{kpc})$ from the nucleus, slightly to the north of the minor axis. The velocity of the molecular gas, $v \sim 1850 \mathrm{~km} \mathrm{~s}^{-1}$, is $\sim 70 \mathrm{kms}^{-1}$ higher than the systemic velocity given by Arnaboldi et al. (1998) and agrees exactly with the stellar velocity derived from absorption line spectra in that region (Fig. 3 of Arnaboldi et al. 1998). Interestingly, beyond $30^{\prime \prime}$ from the nucleus along the minor axis, the stellar radial velocities increase 
on both sides, producing a U-shaped velocity curve, and the velocity dispersion profile is irregular. Arnaboldi et al. argue that this kinematical feature is caused by infalling material within one dynamical time-scale at $40^{\prime \prime}$ (i.e. less than $3 \times 10^{7}$ years ago), since such features are not expected to last longer.

\subsection{Gaseous shells?}

It is interesting to compare NGC 1316 with NGC 5128 (Centaurus A). NGC 5128 lacks tidal tails but it has a more prominent dust lane and a better defined system of shells in both of which atomic and molecular gas have been detected (Eckart et al. 1990; Schiminovich et al. 1994; Charmandaris et al. 2000). NGC 5128 has comparable amounts of $\mathrm{HI}$ and $\mathrm{H}_{2}$ in the region of the dust lane $\left(\sim 3.5 \times 10^{8} M_{\odot}\right)$ whereas NGC 1316 has at least four times more molecular gas than Hi . This difference in Hi content may be due to the fact that the Hi in NGC 1316 is more affected by the X-ray emission, since NGC 1316 is brighter in X-ray than NGC 5128 (factor of 8 in the $0.1-2.4 \mathrm{keV}$ band, ROSAT observations, and factor of 1.4 in the $0.2-$ $4 \mathrm{keV}$ band, Einstein IPC observations). Both galaxies have a double-lobe radio source. The radio distribution, however, has a different orientation: in Centaurus A it is perpendicular to the dust lane whereas in Fornax A it is not. Nuclear effects may therefore affect the gas and dust properties more clearly in Fornax A than in Centaurus A. In the outer parts of NGC 5128, HI is found in the shells, with $\mathrm{CO}$ detected in the highest column density regions with $M(\mathrm{HI}) / M\left(\mathrm{H}_{2}\right) \sim 1$. In NGC 1316 , we could not detect any $\mathrm{CO}$ from the bright outer Hit regions, but our limit on the $\mathrm{H}_{2}$ mass is comparable to the Hi mass. The possible chain of Hi clumps in the outer part of NGC 1316 could be the highest peaks in a continuous shell. More sensitive observations are required to confirm this.

The HST image of NGC 1316 displays beautifully the distribution of the dust (Fig. 11c). Northwest of the nucleus it roughly has a T-shape with a radial extent of about $40^{\prime \prime}$ and an azimuthal extent of almost $90^{\circ}$. CO is detected in the area of both the radial and the azimuthal dust patches. Grillmair et al. (1999) point out the similarity of the radial structure to the photo-evaporating columns in the Eagle Nebula M 16 (Hester et al. 1996), though on a much larger scale, and suggest that it may be due to Rayleigh-Taylor instabilities or subject to erosion by particles or radiation from the nucleus of the galaxy. The azimuthal feature may be a dusty shell. The azimuthal configuration is expected from recently accreted material oscillating between two apocenters and forming shells (e.g. Hernquist \& Quinn 1988). Gaseous shells could form in the same phase-wrapping process if the gas is clumpy and not very dissipative (Charmandaris \& Combes 2000). A detailed dynamical model of the gaseous features in NGC 1316 based on the accretion of a small companion will be the subject of another paper.

\section{Summary and conclusions}

Our new observations of molecular gas (as traced by ${ }^{12} \mathrm{CO}(1-0)$ and ${ }^{12} \mathrm{CO}(2-1)$ line emission) toward the peculiar elliptical galaxy NGC 1316 and a new analysis of Hi data have shown that:

1. About $5 \times 10^{8} M_{\odot}$ of molecular gas is located in the inner $2^{\prime}$ of the galaxy and is mainly associated with the dust patches along the minor axis $\left(\sim 1.8 \times 10^{8} M_{\odot}\right.$ in the southeast, $\sim 10^{8} M_{\odot}$ in the center and $\sim 2.2 \times$ $10^{8} M_{\odot}$ in the northwest). Those mass estimates are based on a Galactic $\mathrm{CO} / \mathrm{H}_{2}$ conversion factor.

2. No neutral atomic gas found was found in the spheroid of NGC 1316 , down to a limit of $\sim 10^{8} M_{\odot}$. Hi was detected at four locations in the outer part of NGC 1316.

3. Two of those locations coincide with bright Hiı regions in a tidal loop. About $2 \times 10^{7} M_{\odot}$ of Hi was found toward the giant Hit region identified by Schweizer (1980) and located 6.7 (or $36.2 \mathrm{kpc}$ ) from the nucleus of NGC 1316. No CO could be detected. About $10^{7} M_{\odot}$ of Hi was detected toward the extended emission line region studied by Mackie \& Fabbiano (1998).

4. The NW molecular gas concentration could be due to recent infall of material onto NGC 1316. It could be a small gas-rich galaxy or the trace of a gaseous shell formed though radial oscillations of infalling material.

5. The two spiral companions of NGC 1316, NGC 1317 to the north and NGC 1310 to the east, are located within the primary beam of the VLA and their Hi distribution and kinematics could be mapped. It was found that NGC 1317 has an unusually small Hi disk that may have been affected by ram-pressure stripping if it passed through the X-ray emitting region of NGC 1316. The Hi distribution of NGC 1310 is asymmetric.

6. There is a hint that the central radio jet of Fornax A is bent owing to interaction with the large concentrations of interstellar gas seen in CO.

Acknowledgements. We are grateful to the SEST staff for their help and support during the observations and to the VLA analysts for their prompt replies and useful help. This work was in part supported by an NSF grant to Columbia University. We have used the tool DEXTER provided by NASA Astrophysics Data System to extract data from Fig. 11a of Schweizer (1980) and from Fig. 2 of Geldzahler \& Fomalont (1984). Those figures and the radio continuum map of Fomalont et al. (1989) shown in Fig. 1 were used with the permission of the authors. We have used images from the Digitized Sky Surveys. The Digitized Sky Surveys were produced at the Space Telescope Science Institute under U.S. Government grant NAG W-2166. The images of these surveys are based on photographic data obtained using the Oschin Schmidt Telescope on Palomar Mountain and the UK Schmidt Telescope. We are grateful to the referee for a careful reading of the manuscript and useful comments.

\section{References}

Arnaboldi, M., Freeman, K. C., Gerhard, O., et al. 1998, ApJ, 507,759 
Arp, H. 1964, ApJ, 139, 1378

Bahcall, J. N., \& Ekers, R. D. 1969, ApJ, 157, 1055

Balcells, M. 1997, ApJ, 486, L87

Balcells, M., van Gorkom, J. H., Sancisi, R., \& del Burgos, C. 2001, AJ, in press

Bosma, A., Smith, R. M., \& Wellington, K. J. 1985, MNRAS, 212,301

Bregman, J. N., Hogg, D. E., \& Roberts, M. S. 1992, ApJ, 387, 484

Brighenti, F., \& Mathews, W. G. 1999, ApJ, 512, 65

Cayatte, V., Kotanyi, C., Balkowski, C., \& van Gorkom, J. H. 1994, AJ, 107, 1003

Charmandaris, V., Combes, F., \& van der Hulst, J. M. 2000, A\&A, 356, L1

Charmandaris, V., \& Combes, F. 2000, in Small Galaxy Groups, IAU Colloq. 174, ed. M. J. Valtonen, \& C. Flynn, 273

Churchwell, E., \& Goss, W. M. 1999, ApJ, 514, 188

da Costa, L. N., Willmer, C. N. A., Pellegrini, P. S., Chaves, O. L., Rité, C., et al. 1998, AJ, 116, 1

Deguchi, S., \& Watson, W. D. 1985, ApJ, 290, 578

de Vaucouleurs, G., de Vaucouleurs, A., Corwin, H. G. Jr. et al. 1991 (RC3), Third Reference Catalogue of Bright Galaxies (Springer-Verlag)

Eckart, A., Cameron, M., Rothermel, H., et al. 1990, A\&A, 363,451

Ekers, R. D., Goss, W. M., Wellington, K. J., et al. 1983, A\&A, 127,361

Fabbiano, G., Kim, D.-W., \& Trinchieri, G. 1992, ApJS, 80, 531

Fabbiano, G., Fassnacht, C., \& Trinchieri, G. 1994, ApJ, 434, 67

Fabbiano, G., \& Schweizer, F. 1995, ApJ, 447, 572

Fomalont, E. B., Ebneter, K. A., van Breugel, W. J. M., \& Ekers, R. D. 1989, ApJ, 346, L17

Galletta, G., Sage, L. J., \& Sparke, L. S. 1997, MNRAS, 284, 773

Geldzahler, B. J., \& Fomalont, E. B. 1984, AJ, 89, 1650

González Delgado, R. M., \& Pérez, E. 2000, MNRAS, 317, 64

Goudfrooij, P., \& Trinchieri, G. 1998, A\&A, 330, 123

Goudfrooij, P., Mack, J., Kissler-Patig, M., Meylan, G., \& Minniti, D. 2001, MNRAS, 322, 643

Grillmair, C. J., Forbes, D. A., Brodie, J. P., \& Elson, R. A. W. 1999, AJ, 117, 167

Haynes, M. P., \& Giovanelli, R. 1984, AJ, 89, 758

Hernquist, L., \& Quinn, P. J. 1988, ApJ, 331, 682

Hernquist, L., \& Spergel, D. N. 1992, ApJ, 399, L117

Hester, J. J., Scowen, P. A., Sankrit, R., et al. 1996, AJ, 11, 2349
Hibbard, J. E., Guhathakurta, P., van Gorkom, J. H., \& Schweizer, F. 1994, AJ, 107, 67

Hibbard, J. E., \& van Gorkom, J. H. 1996, AJ, 111, 655

Horellou, C., Casoli, F., \& Dupraz, C. 1995, A\&A, 303, 361

Huchtmeier, W. K., \& Richter, O.-G. 1989, A General Catalog of Hi Observations of Galaxies (New York: Springer-Verlag)

Kim, D.-W., Fabbiano, G., \& Trinchieri, G. 1992, ApJ, 393, 134

Kim, D.-W., Fabbiano, G., \& Mackie, G. 1998, ApJ, 497, 699

Kuntschner, H. 2000, MNRAS, 315, 184

Loveday, J. 1996, The APM Bright Galaxy Catalogue, MNRAS, 278, 1025

Mackie, G., \& Fabbiano, G. 1998, AJ, 115, 514

Madore, B. F., Freedman, W. L., Silbermann, N., et al. 1999, ApJ, 515, 29

Matthews, T. A., Morgan, W. W., \& Schmidt, M. 1964, ApJ, 140, 35

Quinn, P. J. 1984, ApJ, 279, 596

Sage, L. J., \& Galletta, G. 1993, ApJ, 419, 544

Sansom, A. E., Hibbard, J. E., \& Schweizer, F. 2000, AJ, 120, 1946

Schiminovich, D., van Gorkom, J. H., van der Hulst, J. M., \& Kasow, S. 1994, ApJ, 423, L101

Schiminovich, D., van Gorkom, J. H., van der Hulst, J. M., \& Malin, D. F. 1995, ApJ, 444, L77

Schiminovich, D., van Gorkom, J. H., \& van der Hulst, J. M. 2001 , in prep.

Schulman, E., \& Fomalont, E. B., 1992, AJ, 103, 1138

Schweizer, F. 1980, ApJ, 237, 303 (S80)

Schweizer, F. 1981, ApJ, 246, 722

Schweizer, F., \& Seitzer, P. 1998, AJ, 116, 2209

Shaya, E. J., Dowling, D. M., Currie, D. G., et al. 1996, AJ, 111,2212

Sofue, Y., Wakamatsu, K., Taniguchi, Y., \& Nakai, N. 1993, PASP, 45, 43

Strong, A. W., Bloemen, J. B. G. M., Dame, T. M., et al. 1988, A\&A, 207, 1

van Gorkom, J. H., Schiminovich, D., in The Second Stromlo Symposium: The Nature of Elliptical Galaxies, ASP Conf. Ser. 116, ed. M. Arnaboldi, G. S. da Costa, \& P. Saha, 310

Wade, C. M. 1961, Publ. NRAO, 1, 99

Wang, Z., Schweizer, F., \& Scoville, N. Z. 1992, ApJ, 396, 510

Wiklind, T., \& Henkel, C. 1989, A\&A, 225, 1

Wiklind, T., Combes, F., \& Henkel, C. 1995, A\&A, 297, 643

Wozniak, H., Friedli, D., Martinet, L., et al. 1995, A\&AS, 111, 115 